\title{
Gradhiva
}

GRADHIV

Revue d'anthropologie et d'histoire des arts

26 | 2017

En croire ses sens

\section{Odes à l'art de la côte Nord-Ouest. Surréalisme et ethnographie}

Odes to Northwest Coast Art.Surrealism and Ethnography

\section{Marie Mauzé}

\section{(2) OpenEdition}

1 Journals

Édition électronique

URL : http://journals.openedition.org/gradhiva/3467

DOI : 10.4000/gradhiva.3467

ISSN : 1760-849X

Éditeur

Musée du quai Branly Jacques Chirac

\section{Édition imprimée}

Date de publication : 6 décembre 2017

Pagination : 180-209

ISBN : 978-2-35744-096-8

ISSN : 0764-8928

\section{Référence électronique}

Marie Mauzé, «Odes à l'art de la côte Nord-Ouest. Surréalisme et ethnographie », Gradhiva [En ligne], 26 | 2017, mis en ligne le 06 décembre 2019, consulté le 03 janvier 2020. URL : http://

journals.openedition.org/gradhiva/3467 ; DOI : 10.4000/gradhiva.3467

(c) musée du quai Branly 


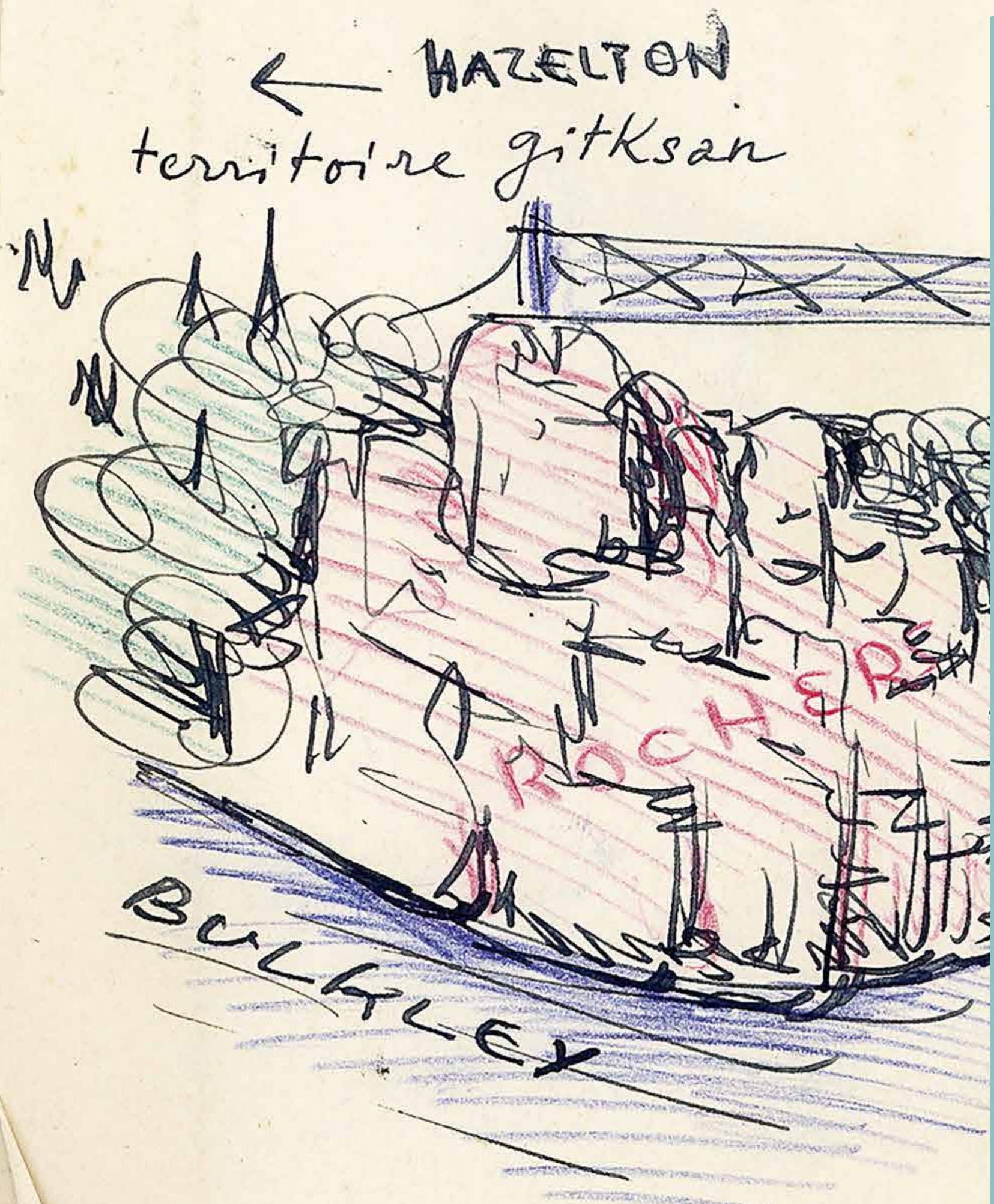

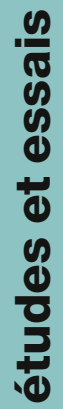




\section{Odes à l'art de la côte Nord-Ouest 1}

\section{Surréalisme et ethnographie}

par Marie Mauzé

Cet article se propose d'examiner les rapports entre surréalisme et ethnographie à travers une série d'articles sur l'art et la culture de la côte Nord-Ouest (Amérique du Nord) publiés par les peintres Kurt Seligmann et Wolfgang Paalen, le critique d'art Georges Duthuit et André Breton, entre 1938 et 1950, à une époque où les surréalistes étaient reconnus pour leur expertise en matière d'art primitif. Une lecture attentive de ces textes montre l'étendue et la diversité du savoir ethnographique de leurs auteurs en dépit de leur absence de rigueur dans le traitement des faits ethnographiques à des fins poétiques et esthétiques. Critiqués par les ethnologues, les surréalistes se sont néanmoins interrogés avant eux sur le statut du mythe et de la parole comme mode d'expression littéraire dans les sociétés non occidentales ou encore sur les rapports étroits entre croyance au surnaturel et émotion esthétique.
1. Ce texte est une version

entièrement remaniée

d'une communication

présentée au colloque

international « Inventions

et réinventions des arts

primitifs " organisé

par le département

de la recherche

et de l'enseignement

du musée du quai Branly

et l'université

Paris I-Panthéon-

Sorbonne, en septembre

2013. Je remercie les

évaluateurs de ce texte

ainsi que Jacqueline

Ancelot, Michèle Coquet

et Michèle Therrien

pour leurs commentaires. 


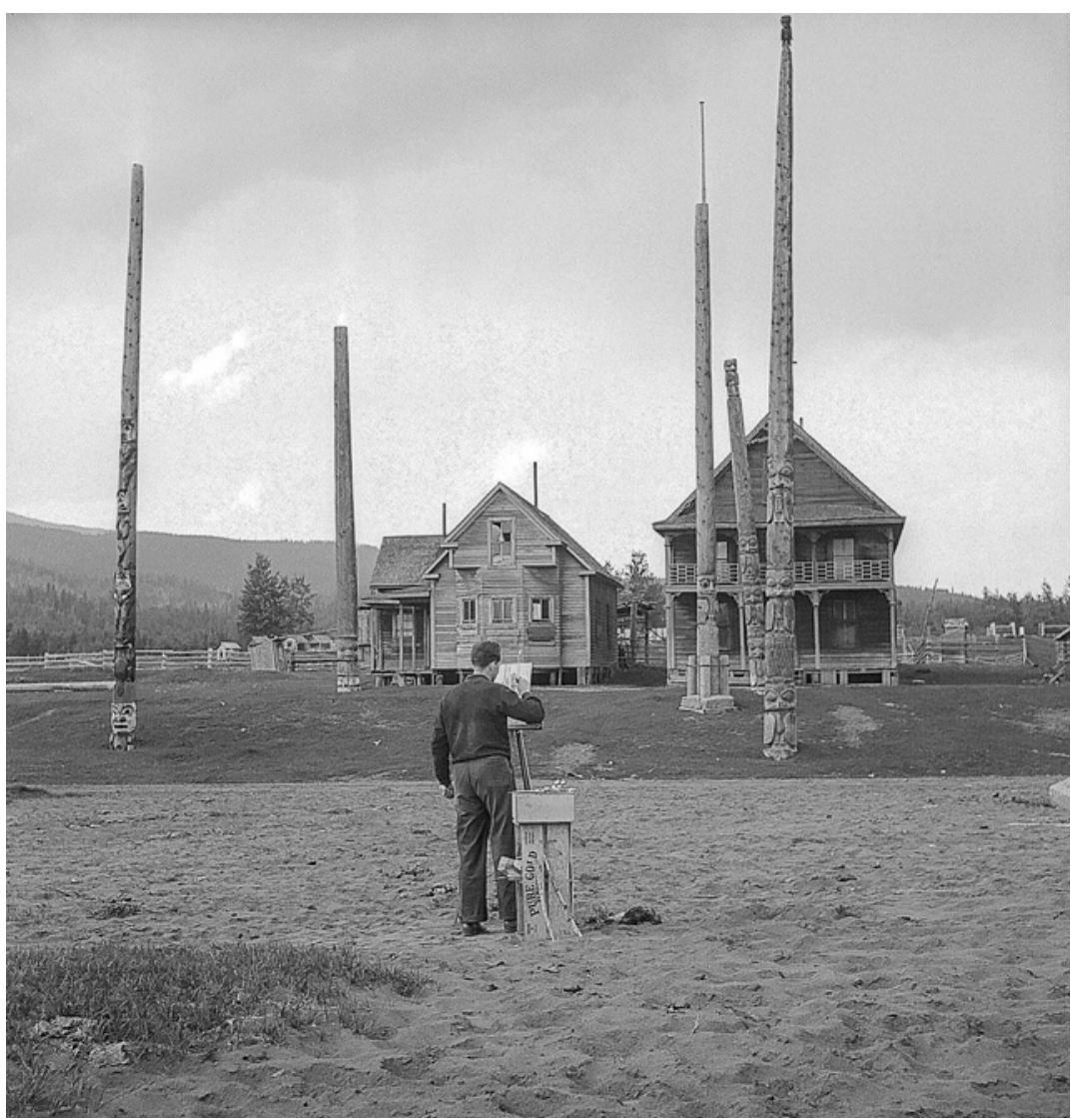


En quête d'une nouvelle sensibilité propre à leur permettre de dépasser les cadres d'un environnement intellectuel qu'ils jugeaient stérile au lendemain de la Grande Guerre, les surréalistes se sont intéressés aux sociétés non-occidentales. L'ethnographie leur révélait une infinie variété de modèles sociaux et de systèmes de croyance qui leur ouvraient des horizons nouveaux et fournissaient la matière à nourrir une réflexion critique sur la civilisation occidentale $^{2}$. Comme le souligne Alfred Métraux (Métraux et Bing 1964: 21), les surréalistes ont puisé dans les textes ethnographiques des éléments grâce auxquels ils ont pu construire une nouvelle philosophie: «Brusquement, les peuples exotiques venaient confirmer, en quelque sorte, l'existence d'aspirations qui ne pouvaient s'affirmer dans notre propre civilisation. » Pour faire rempart au rationalisme occidental, ils cherchaient «tout ce qu'il y avait d'incongru, d'extraordinaire, dans ces civilisations exotiques». (Ibid.)

À la lecture des travaux de James George Frazer et de Lucien Lévy-Bruhl, ils se familiarisent avec la «pensée primitive» et exaltent certains traits dont ils pensent qu'ils en constituent l'armature; en accordant une place de choix au rêve, à la magie, au mythe et à l'inconscient libérateur de la puissance créatrice, les théories ethnologiques et psychologiques à l'honneur à l'époque, ils épousent celles qui font écho à leurs préoccupations.

L'objectif de cet article est moins de traiter de la provenance des collections d'art amérindien réunies par les surréalistes à partir des années 1920, ou des relations que ceux-ci entretenaient avec les objets, que d'étudier les rapports complexes - voire paradoxaux - entre surréalisme et ethnographie ${ }^{3}$ en se fondant sur leur traitement des matériaux de la côte Nord-Ouest de l'Amérique du Nord. Rares sont les écrits des surréalistes sur l'art et la culture des peuples amérindiens et yup'ik, sauf quelques fragments de poèmes ou propos allusifs se rapportant à cet univers dans les entretiens ou la correspondance. Amateurs d'art amérindien, ni Roberto Matta, ni Max Ernst, ni Robert Lebel, ni Enrico Donati, ni Isabelle Waldberg n'ont laissé de témoignage substantiel sur leurs rapports à cet art. L'étude, ancrée dans une démarche anthropologique, s'appuiera sur l'analyse d'un corpus de textes signés Kurt Seligmann (1900-1962), Wolfgang Paalen (1905-1959) - deux peintres qui ont rejoint le mouvement surréaliste dans les années 1930 et ont séjourné dans le Nord-Ouest -, Georges Duthuit (1891-1973) - critique d'art qui fit partie, à New York, de la constellation surréaliste dans les années 1940 - et André Breton (1896-1966), le poète et fondateur du mouvement surréaliste. Cette étude fera apparaître l'étendue du savoir ethnographique de ces auteurs, utilisé au service d'un imaginaire poétique et d'une réflexion sur l'état de l'art moderne et de ses modalités de représentation.

Ces textes, qui célèbrent la richesse des cultures de la côte Nord-Ouest et la grandeur de l'art de cette région, montrent qu'au-delà de la relation sensible à l'objet, leurs auteurs avaient sur ces cultures une véritable connaissance qu'ils avaient acquise à la faveur de la fréquentation des musées d'ethnographie, de virées chez les antiquaires et bien évidemment de lectures. Si certains d'entre eux avaient pu se procurer quelques rapports annuels de la Smithsonian Institution dans les années 1920 (Cowling 1978: 487), nul doute que leur fonds ethnographique s'est enrichi lors de leur séjour à New York où les volumes du Bureau of American Ethnology étaient plus accessibles qu'en Europe ${ }^{4}$. En outre, l'amitié que Breton et ses compagnons avaient nouée avec Claude Lévi-Strauss avait probablement stimulé leur curiosité.
2. Voir Bédouin 1961; Blachère 1996; Cowling 1978 ; Maurer 1991.

3. Cette question a été abordée par Blachère (1996) principalement à partir de l'examen de matériaux océaniens.

4. Pour l'inventaire du fonds ethnologique des bibliothèques de Breton et Paalen, voir Breton 2003: 40-41; Winter 1992 : 21 ; 2003 : 18-21. 
5. Galeriste et éditeur de la Gazette des beaux-arts.

6. Seligmann n'a jamais rencontré Barbeau mais il connaissait son rôle dans l'acquisition du mât par le musée.

7. Les Wetsuwet'en sont les voisins des Gitksan (Tsimshian).

\section{ci-contre}

fig. 2

Mâts totémiques d'Anklawrh et de

Gyaedem-skanees, Hagwilget, Colombie britannique, 1923. Musée canadien de I'histoire, fonds Marius Barbeau, 59526.
Quelques-uns d'entre eux, Robert et Nina Lebel et Isabelle Waldberg, assistaient aux cours qu'il dispensait en 1942-1944 à l'École libre des hautes études (Waldberg et Waldberg 1992: 202).

Témoignant d'une grande érudition, les textes de ces quatre auteurs rédigés entre 1938 et 1950 appartiennent à un genre singulier. Réunissant dans un même mouvement une certaine exigence du savoir ethnologique et la saveur d'une sensibilité poétique, ils sont en cela caractéristiques de ce que William Rubin (1991: 10) définit comme un primitivisme «intellectualisé » qui emprunte explicitement ses références aux peuples non-occidentaux, ici, pour exalter un idéal surréaliste. Ces textes font état d'une fascination partagée pour les arts du Pacifique Nord-Ouest. On y observe des thèmes communs: la mythologie, le totémisme et les mâts-totems, les masques à transformation, le potlatch et, dans un registre politique, l'effet délétère de la colonisation sur les cultures autochtones. Toutefois, chaque auteur propose un éclairage différent, ce qui s'explique par une expérience individuelle des objets, l'expression d'une sensibilité propre, mais aussi par la nature du projet qui sous-tend l'écriture de ces textes: témoignage ethnographique pour Seligmann, réflexions sur les conditions d'émergence d'un nouvel art moderne et statut de l'artiste pour Paalen et excursions poétiques pour Duthuit et Breton où sont notamment exprimées des interrogations sur l'efficacité de la performance rituelle pour l'un et l'importance de la mascarade pour l'autre.

\section{Kurt Seligmann: une expérience d'ethnographe}

Peintre d'origine suisse et neveu par alliance de Georges Wildenstein $\mathbf{5}$, Seligmann partage avec les surréalistes un commun intérêt pour l'ethnologie et les arts amérindiens. Au cours de sa vie, il développe également une curiosité pour la mythologie, les sciences occultes et les pratiques magiques. En 1934, il découvre dans Cahiers d'art l'article «Sculptures en bois de l'Amérique $\mathrm{N}-\mathrm{O}$ » de Leonhard Adam, auteur d'un ouvrage sur l'art de la côte Nord-Ouest paru en 1923 (Hauser 1997: 147). Dans cet article, l'importance est donnée aux sculptures monumentales exposées dans la galerie de la côte Nord-Ouest de l'American Museum of Natural History, photographiées en plan moyen ou gros plan; le texte se résume quasiment aux légendes où l'auteur identifie l'origine des sculptures ainsi que les personnages représentés. Cette publication aurait frappé Seligmann et suscité l'envie de se rendre sur place pour rapporter un «totem » en Europe. Immédiatement après, il lit Totémisme et Exogamie de Frazer (ibid.).

C'est muni d'un bagage fortement ancré dans l'ethnologie du tournant du $x x^{e}$ siècle, amalgamant animisme et totémisme, magie et religion, que Seligmann entreprend un voyage sur la côte Nord-Ouest en juin 1938, accompagné de son épouse Arlette. II est le premier artiste surréaliste à tenter l'aventure, imité par Paalen l'été suivant. Seligmann connaissait l'ouvrage de Marius Barbeau (1929), Totem Poles of the Gitksan, qui a pu lui servir de guide dans la vallée de la Skeena. Le livre offre un inventaire - avec notices et photographies à l'appui - de quelque cinquante-deux mâts héraldiques de cette région, effectué entre 1920 et 1926 en vue de leur préservation. Barbeau n'est pas un inconnu au musée de l'Homme. Par son intermédiaire, le musée reçoit, en 1930, le mât dit de l'Ours, offert par les chemins de fer canadiens ${ }^{6}$. Avec l'appui moral du musée (Sawin 1995: 23), Seligmann fait l'acquisition d'un mât héraldique wetsuwet'en ${ }^{7}$ qui sera érigé devant 


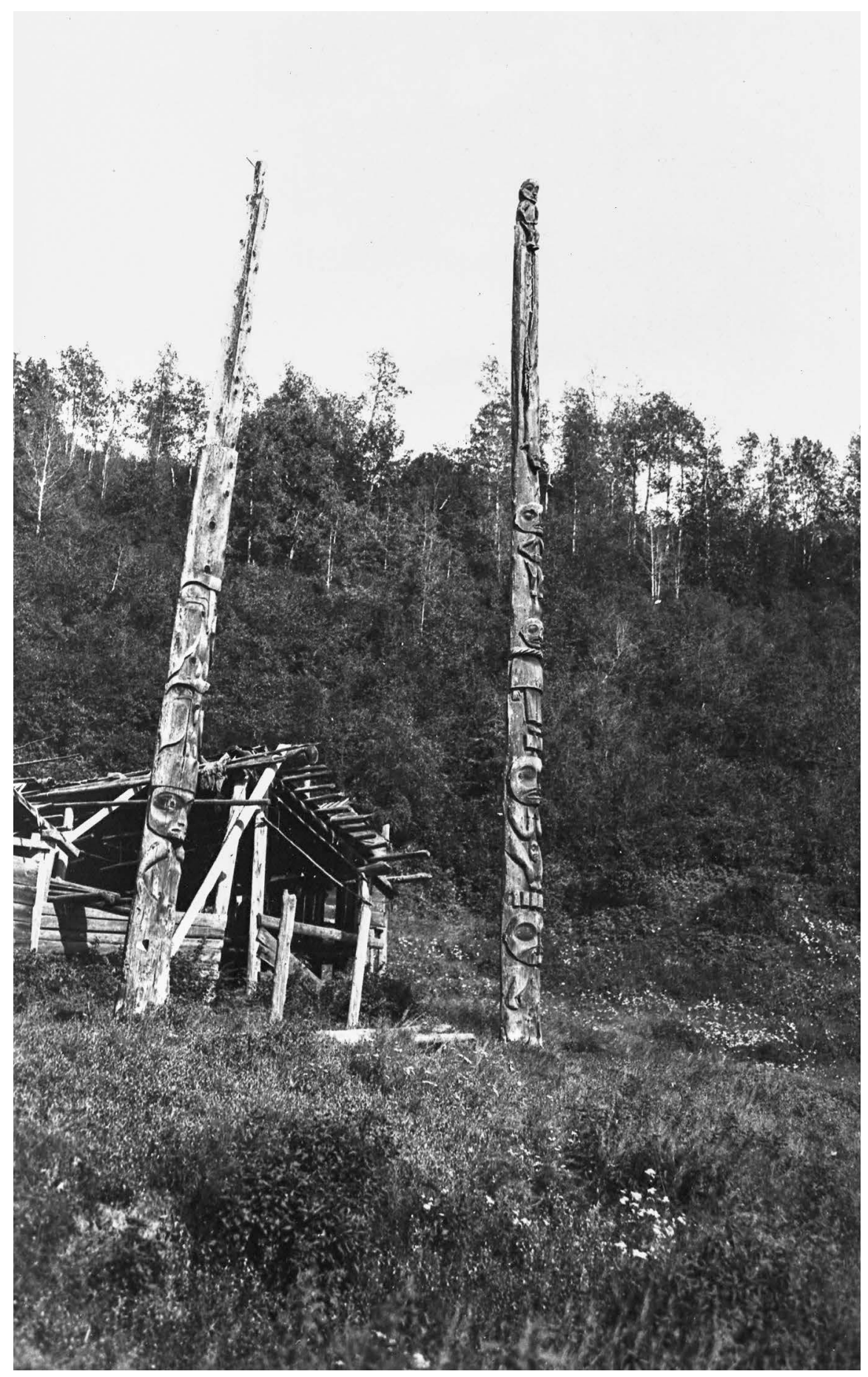




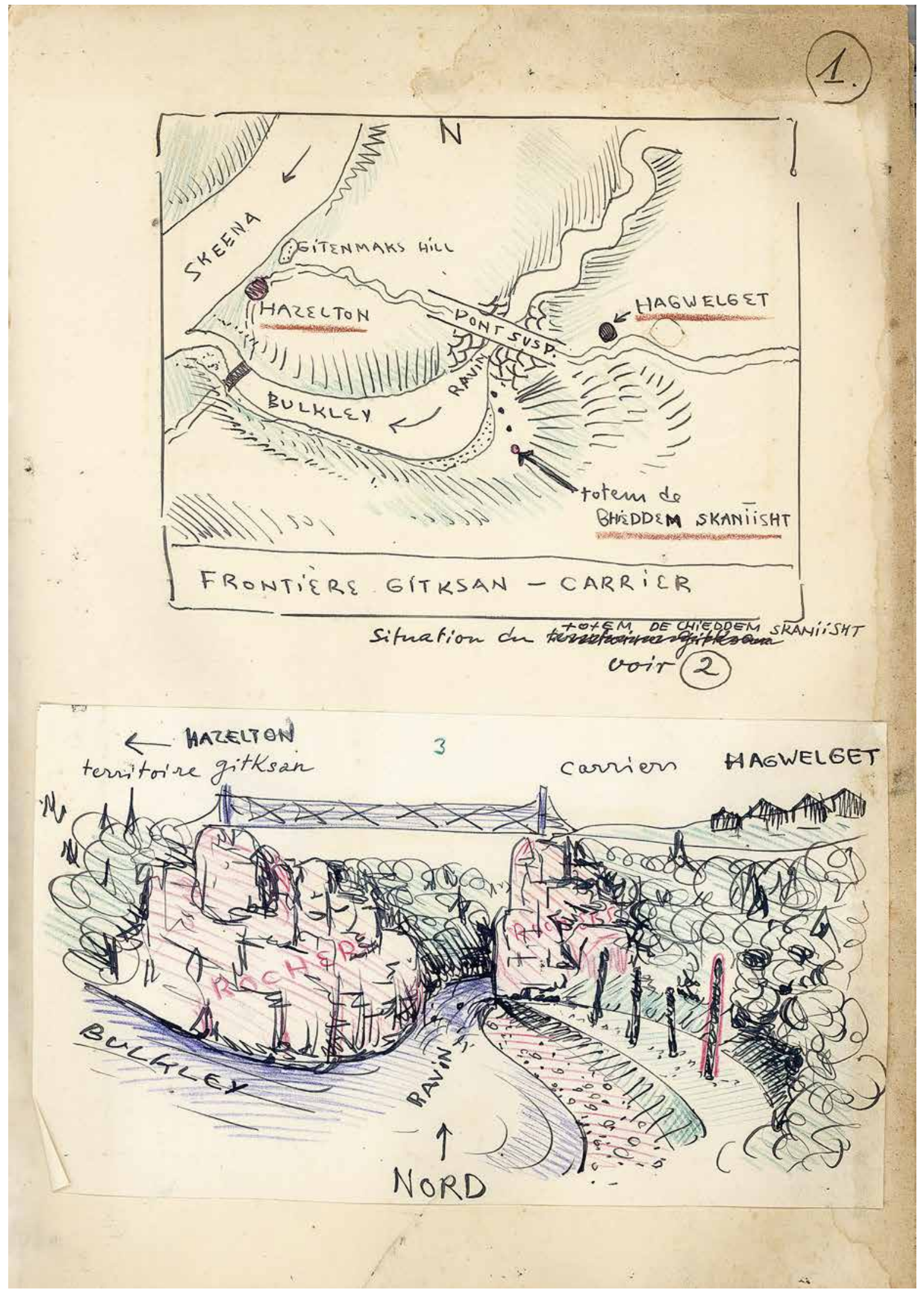


l'établissement en janvier 1939, et à propos duquel Benjamin Péret (1939) écrit dans le Paris-Soir du 21 janvier: “Clou du Musée de l'Homme et inauguré hier soir. Krikiett, le plus haut totem d'Europe ${ }^{\mathbf{8}}$.»

Durant son séjour d'environ quatre mois dans le village de Hazelton, Seligmann n'abandonne pas sa pratique artistique (Hauser 1997) mais se consacre principalement à la tâche qu'il s'est assignée: l'acquisition d'un mât. L'entreprise n'est pas chose facile à cette époque car outre le moral affaibli des communautés autochtones auxquelles le gouvernement canadien avait interdit toute activité cérémonielle, leurs représentants s'opposaient vivement au programme de sauvegarde consistant à extraire les mâts héraldiques de leur lieu d'origine pour les relocaliser le long du tracé de la voie de chemin de fer reliant Edmonton à Prince Rupert. Les mâts devaient être admirés par les touristes moins comme des vestiges d'une culture disparue que comme partie intégrante du patrimoine culturel canadien (Dawn 2006). D'une manière générale, les chefs gitksan étaient réticents à se séparer de ces sculptures monumentales car les blasons figurés établissaient un lien indissociable entre les groupes sociaux, leur histoire et leur territoire.

Seligmann porte son choix sur le plus ancien, le mieux conservé et le plus habilement sculpté parmi les quatre mâts encore debout dans le village abandonné d'Hagwilget (1939b: 124); il n'en fera l'acquisition pour la somme de 100 dollars canadiens qu'au terme de cinq semaines de négociations avec ceux qui prétendent détenir sur lui un droit de propriété. Pour conjurer les événements dramatiques survenus dans la communauté après l'accord scellé avec les différents partenaires - la foudre abat un des quatre mâts; un chasseur est attaqué par un ours - il est adopté au sein du clan propriétaire du mât grâce à la célébration de son mariage fictif avec une femme décédée (Péret 1939; Hauser 1997: 149).

Sur le terrain, Seligmann se comporte en ethnologue. II conduit des entretiens, collecte des mythes et des légendes et se documente sur l'histoire locale. On lui doit une carte montrant la situation géographique du village d'Hagwilget (Mauzé 2008) et la localisation du mât héraldique. II est l'auteur, avec sa femme, d'un film et d'un grand nombre de photographies de mâts héraldiques prises in situ dans plusieurs villages de la vallée de la Skeena ainsi que de clichés représentant les protagonistes de la transaction.

II publie quatre textes avec la mythologie pour thème commun, dont trois rendent compte des données recueillies sur le terrain. L'article paru dans le Journal des Américanistes en 1939 s'apparente à une monographie dans laquelle l'ethnographe amateur fournit les informations nécessaires pour comprendre l'histoire du mât, les circonstances de son acquisition, les raisons du choix ${ }^{9}$. Ainsi, il rapporte le mythe qui lui est associé où il est question du rapt d'une femme par le géant cannibale Kaiget, de la vengeance du fils de la victime qui extermine les habitants du village, puis de sa mort dans les flammes. Kaiget, personnage central du mythe adopté comme blason par les ancêtres de Gedem Skanees, est figuré sur le mât sous trois apparences différentes.

Dans cet article bien documenté, Seligmann évoque aussi les questions de migration, d'emprunts culturels, de style et de composition des figures représentées sur le mât, thèmes traités une décennie auparavant par Barbeau. Auprès de ce dernier, il vérifie, par correspondance, l'exactitude de ses
8. Cette sculpture monumentale se dresse aujourd'hui dans le hall du musée du quai Branly.

9. Il est attiré par la "belle patine qui varie du gris argent au brun cuivré " (Seligmann 1939b: 126). 
10. Lettre de Seligmann à Barbeau (décembre 1938); lettre de Barbeau à Seligmann (20 janvier 1939); lettre de Seligmann à Barbeau (25 février 1939), fonds Marius Barbeau, correspondance, dossier "Seligmann, Kurt". boîte B238f73.

11. Journal de la Société des Américanistes, 31, 1939: XXI, XVII.

12. Manuscrit, fonds Kurt Seligmann (VII-C-103 M), Musée canadien de l'histoire.

\section{ci-contre}

fig. 4

Kurt Seligmann examinant un mât héraldique dans le village de Gitenmaks Skeena River, 1938.

Avec l'aimable autorisation de The Seligmann Center at the Orange County

Citizens Foundation. propres données ${ }^{\mathbf{1 0}}$. Fruit du travail d'un artiste, sa recherche, sérieuse, trouve légitimement sa place dans la revue de la Société des Américanistes, dont Seligmann devient membre en février 1939, trois mois avant Paalen ${ }^{\mathbf{1 1}}$.

Les trois autres textes sont accueillis dans $x x^{e}$ siècle et Minotaure. Reprenant les poncifs de l'époque pour laquelle le totémisme renvoyait à un premier stade de l'humanité où hommes et animaux étaient indistincts, il établit dans $x x^{e}$ siècle (Seligmann 1938) une comparaison entre les mythologies de la côte Nord-Ouest et cinghalaise qui ont, selon lui, une "signification totémique». L'année suivante, il fait paraître la transcription de la légende du mât Kryegett (sic) telle qu'elle lui a été racontée par le chef gitksan Donald Grey. Dans son «Entretien avec un Tsimshian » publié dans Minotaure en 1939, Seligmann restitue un échange avec Grey sur la comparaison entre les mythes européens et amérindiens, thème qui l'intéressait au plus haut point, passionné qu'il était de mythologie, source d'inspiration de bon nombre de ses œuvres (Seligmann 1939c).

Les textes cités révèlent la fibre ethnographique de Seligmann, mais on reconnaît dans un manuscrit d'une vingtaine de pages, contenant des notes de lecture et des remarques personnelles ${ }^{\mathbf{1 2}}$, un observateur attentif à la situation sociale et politique des sociétés amérindiennes, inscrivant sa réflexion dans le temps présent. D'un côté, il interprète l'état de délabrement des mâts comme la métaphore de la déliquescence des cultures autochtones sous les effets pervers de la colonisation canadienne qui précipite les protagonistes à leur propre perte en brûlant eux-mêmes leurs sculptures, la jeune génération délaissant la tradition pour la modernité, où opère «la magie des bicyclettes, des cinémas et des chemins de fer» (ibid. : 67). De l'autre, dans ledit manuscrit, il perçoit dans la création de deux nouveaux mâts l'expression encourageante d'une forme de résistance au pouvoir colonial:

Bien que pratiquement éteint, l'art de sculpter les totems meurt difficilement. Après avoir perdu une grande partie de leurs coutumes, après avoir vu se dissoudre leur organisation sociale et leurs croyances, vivant sans raison d'être et sans pouvoir s'adapter à leur nouvelle vie, les Indiens s'accrochent désespérément aux totems, témoins de leur ancienne «splendeur». Deux d'entre eux ont fait sculpter récemment des mâts totémiques.

On devine chez Seligmann une véritable compréhension de la logique à l'œuvre au sein des sociétés de la côte Nord-Ouest par rapport à la préservation de mâts héraldiques. Ayant admis le processus naturel de leur désintégration en raison de la nature des matériaux dont ils sont faits - processus biologique à l'image de la dégradation du corps humain -, il doute du bienfondé de la restauration du mât qu'il a collecté sur place; il admet qu'«une fois debout, [le mât] ne doit plus être modifié ou touché; même si par l'âge, la moisissure, il est menacé de destruction, on le laisse tomber et pourrir. Une fois couché, poursuit-il, il a perdu sa force magique, il est considéré comme mort, tel qu'un être humain, et les sentiments à son égard sont adéquats; il est bientôt oublié » (ibid.). Seligmann fait sienne l'idée que le mât héraldique revêt sa pleine signification sociale et politique lors de son installation, suivie de la cérémonie de distribution de biens validant les revendications familiales et territoriales d'un chef qui sont représentées par les blasons décrits dans les mythes et figurés sur le mât. Une fois érigé, le mât a 


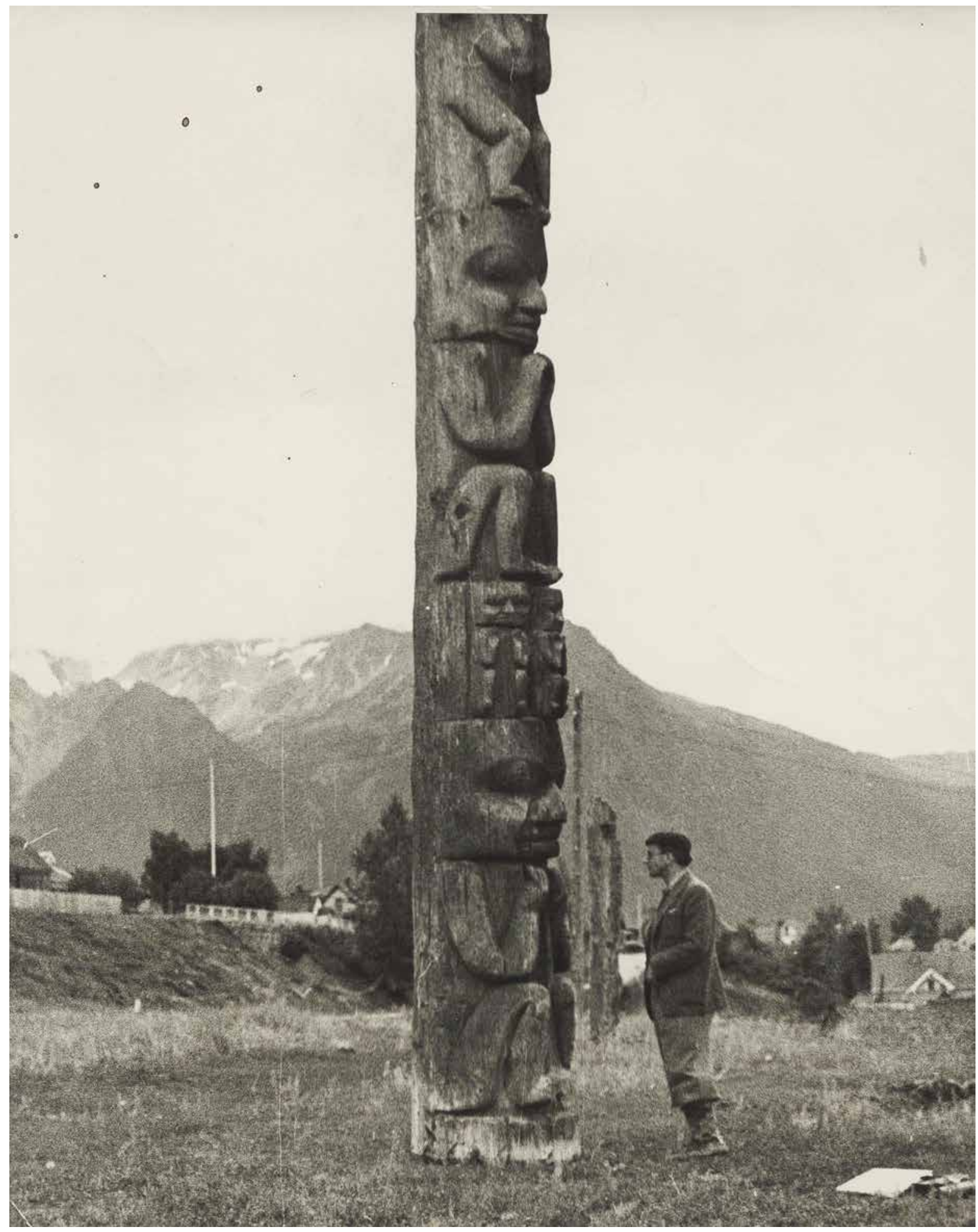


13. À Breton, il écrit: "Réellement partis pour le Mexique, toutes dispositions prises pour y aller de New York, l'ancien [sic] envie de voir ces régions d'affinités plus qu'électives l'emportait totalement. J'ai intensément regardé et vu! » Lettre de Paalen à Breton, 21 août 1939, bibliothèque Doucet, BRTC 2232.

14. La couverture du numéro spécial (4-5) de Dyn est illustrée par un dessin acheté à l'artiste kwakwaka'wakw Henry Speck (1909-1971). accompli sa fonction: le droit à des prérogatives ayant été reconnu, peu importe la pérennité de l'objet qui est censé incarner ce droit. Alors se pose la question de la pertinence de la collecte du mât Kaiget pour le musée de l'Homme. Quel est son statut? Un témoignage de «l'ancienne splendeur» des cultures de la côte Nord-Ouest?

Seligmann était bien décidé à s'acquitter de l'engagement qu'il avait envers le musée de collecter une sculpture monumentale. Si l'on en croit la presse qui le salua comme un héros ayant contribué au sauvetage d'une sculpture (Meunier 2008: 88), il s'agissait presque d'un trophée. La démarche de Seligmann rejoint celle des ethnologues de l'époque qui collectaient des objets-témoins de cultures menacées de disparition et réunissaient les données relatives à leur origine, leur contexte d'utilisation et leur signification, éléments que seule la pratique de terrain permet de recueillir. L'apprentissage du métier d'ethnographe fut pour l'artiste une expérience de courte durée; elle est unique dans l'histoire des relations entre surréalisme et ethnographie (Mauzé 2008).

\section{Wolfgang Paalen: l'ethnographie à l'épreuve de l'art}

Au cours de l'été 1939, le peintre d'origine autrichienne Wolfgang Paalen voyage, en compagnie de sa femme, la peintre et poétesse Alice Rahon, et de son amie, la photographe Eva Sulzer, en Colombie britannique et en Alaska. II y a urgence à quitter la France, écrit-il «en cet été sourd de 1939" (Paalen, 1942b: 46; 1994a: 35). Le projet initial était de se rendre depuis New York au Mexique à l'invitation de Frieda Kahlo et Diego Rivera. La décision d'ajourner de trois mois l'arrivée à Mexico et de faire un long détour par le Nord-Ouest semble avoir été motivée par l'envie impérieuse de voir «autrement qu'en rêve, les grandes forêts de l'Amérique du Nord " (Paalen 1942c: 45 ; 1994a: 39) et de nouer un contact immédiat avec les peuples de la côte Nord-Ouest dont il admire l'art et la mythologie ${ }^{13}$. II s'était déjà familiarisé avec l'art de la région lors de ses visites dans les musées d'ethnographie de Vienne et de Berlin, bien avant sa rencontre avec les surréalistes à Paris (Winter 2003: xxIv-xxv).

Avant de quitter Paris, Paalen s'est assuré le soutien du musée de l'Homme afin de prendre contact avec le ministère des Affaires indiennes à Ottawa où il obtient, au titre de représentant du musée français, l'autorisation de visiter les réserves indiennes de la Colombie britannique (Winter 1992: 21). C'est avec ses interlocuteurs canadiens du musée national qu'il définit son itinéraire (Paalen 1994b: 13). Il s'arrête dans de nombreuses localités sans prendre le temps, contrairement à Seligmann, de s'installer dans un lieu particulier. En Colombie britannique, il se rend en territoire gitksan et tsimshian, tlingit, haida, nuxalk et kwakwaka'wakw. Les trois voyageurs font une escapade à Wrangell et Sitka, en Alaska. Paalen passe plusieurs jours dans les Haida Gwaii (îles de la Reine-Charlotte) en compagnie de Barbeau avec lequel il visite quelques sites abandonnés au nord et au sud de l'archipel, là où subsistent des vestiges de mâts héraldiques (Barbeau, s. d: 582, 585). À l'opposé de Seligmann, Paalen ne semble pas avoir noué des liens étroits avec les autochtones qu'il a croisés sur son chemin. II n'en évoque précisément ni les lieux de résidence ni les noms, alors même que des témoignages indiquent qu'il a rencontré des artistes haida et kwakwaka'wakw, comme l'attestent les mémoires de Barbeau ${ }^{14}$. En revanche, il s'adonne à la 
collecte d'objets «authentiques » qu'il acquiert dans les boutiques de curios ou dans les villages, si bien qu'il n'a aucun scrupule à acquérir des masques pillés sous ses yeux dans la tombe d'un chamane (Paalen 1994b: 31; 1943: $19,36)^{15}$. II y trouve là l'assurance de leur ancienneté et de leur authenticité que confirmait leur utilisation rituelle. Les pièces rassemblées sur place reflètent la vibrante expression de sociétés traditionnelles, mais des signes de la décadence artistique lui apparaissent également, qu'il s'agisse des objets de mauvaise facture fabriqués à des fins commerciales ou de misérables mâts qu'il découvre dans le village tlingit de Ketchikan en Alaska: «Forêt magnifiquement sauvage, gâchée au début par d'affreux poteaux totémiques neufs, laids comme des quilles. » (Paalen 1994b: 23) C'est en esthète primitiviste et non en ethnographe qu'il apprécie l'art de la région, ne recueillant aucune donnée sur les pièces qu'il a collectées. Pour autant, il a une perception aigüe des conditions faites par le pouvoir colonial aux cultures autochtones, avec l'interdiction du potlatch et l'impossibilité pour les artistes de créer des objets à usage rituel (Paalen 1943: 18).

Dans une lettre à Breton en août 1939, il exprime son sentiment de tristesse face à ces cultures qui disparaissent englouties dans une nature plus forte qu'elles, qui les absorbe inexorablement:

Quelques documentations, quelques trouvailles et, je pense, des aperçus nouveaux sur cet art, infiniment plus grand que tout ce qu'on peut en supposer en Europe, le sentiment d'une longue marche dans une pénombre plus profonde que celle de la forêt, qui permettrait juste d'apercevoir le dernier rayon d'une culture des plus étrangements [sic] splendide dans une nature inexpugnablement sauvage - et nous voilà au terme de notre parcours du Nord-Ouest ${ }^{16}$.

C'est sous le signe du terme «totem» que Paalen a entrepris son périple, comme le montrent ses écrits publiés dans Dyn: «Paysage totémique », paru en quatre livraisons, et illustré par les photographies d'Eva Sulzer ${ }^{17}$, puis «Totem Art». Les deux textes ont une ambition différente. Le premier, publié sous forme de "fragments", tient de la méditation philosophique sur la rencontre de l'artiste avec le Nord-Ouest où l'immense force des «terres du silence», la grandeur de l'art «totémique» et l'omniprésence du mythe sont confondus dans une quête mystique et esthétique. II tient pour acquise l'existence d'un lien indissociable entre le paysage et les peuples qui l'habitent, ce qui est donné à voir notamment dans l'art qui révèle son ancrage dans un lieu spécifique. L'expression artistique la plus emblématique y est représentée par les sculptures monumentales indiscernables des arbres qui les entourent, manifestation remarquable d'une fusion de l'art et la nature correspondant à sa propre conception du paysage totémique ${ }^{\mathbf{1 8}}$ proche de la notion romantique de paysage, associée à celle de ruine (Dickson 2014):

Sous les inexpugnables voûtes de sa forêt sans âge toujours débordantes d'ours, d'aigles et de loups, se cachent les épaves scorifiées d'une culture des plus étranges faite par des hommes à face de lune et de cuivre. (1942 b: 46)
15. Voir aussi Winter 1992: 20; 2003: 162; Mauzé 2008: 9-10.

16. Lettre de Paalen à Breton, 21 août 1939, bibliothèque Doucet, BRTC2232.

17. Aux trois cents photographies prises par Sulzer, il faut ajouter dix bobines de pellicule relatant le voyage (Neufert 2011).

18. Voir Paalen 1942c: 45-46; 1994a : 39-41. Voir aussi Mauzé 2008 et Dickson 2014 sur la notion de "paysage totémique " chez Paalen. 

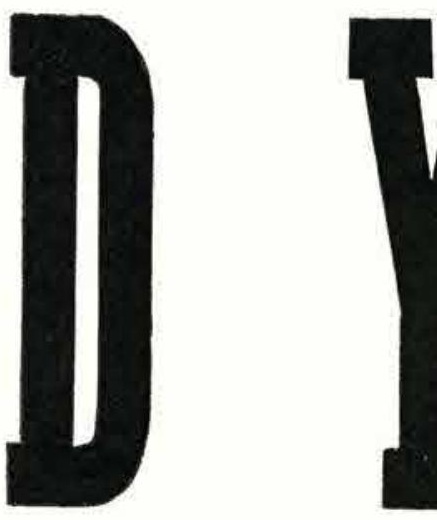

4.

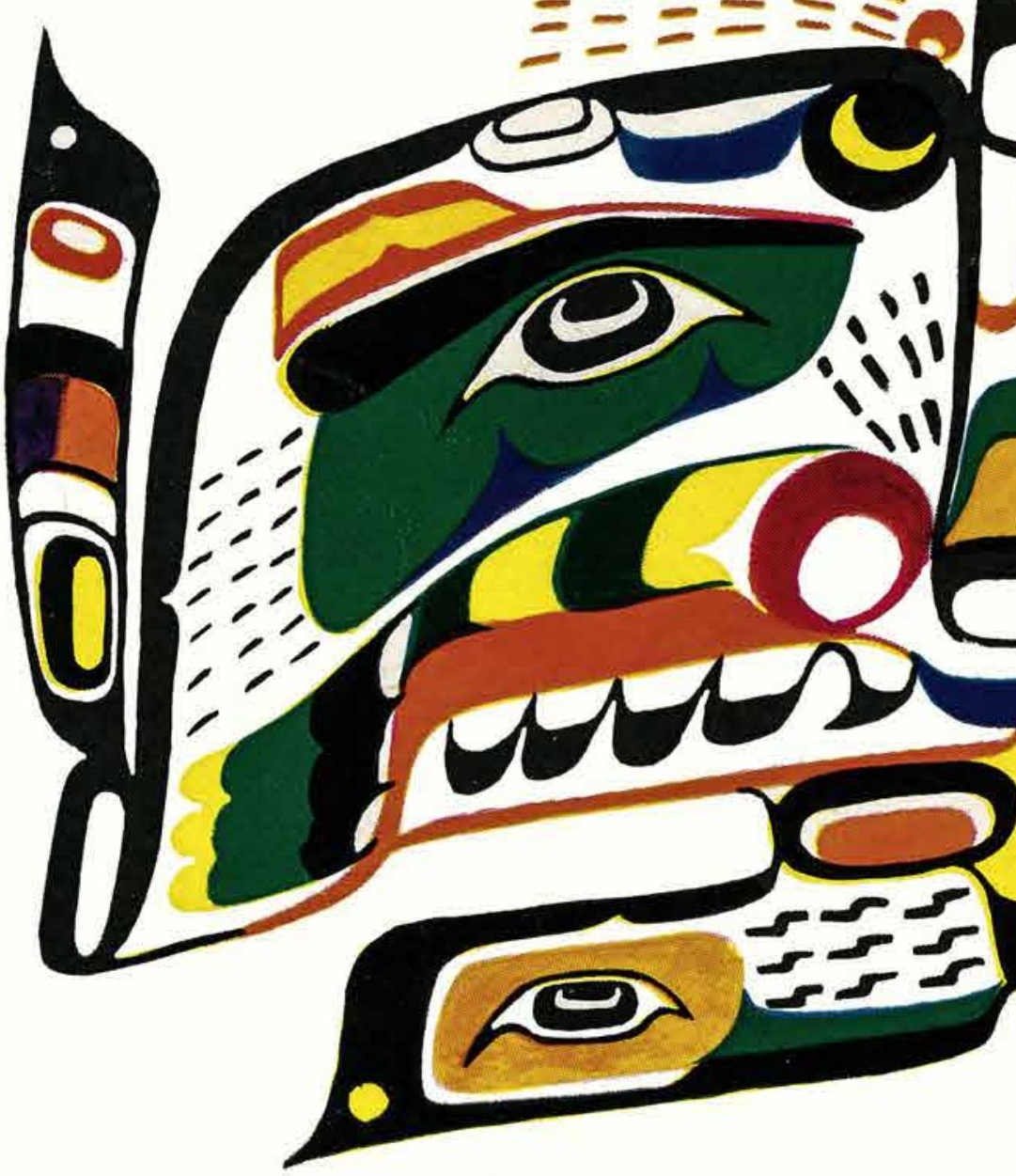


19. Voir préface au numéro Dyn 1 (s. p.) ou Paalen $1943: 36$

20. Nombre des illustrations publiées dans «Totem Art » sont reprises dans le catalogue de l'exposition «El Arte indígena de Norte América " organisée au Musée national d'anthropologie et d'histoire à Mexico en 1945.

\section{double page précédente}

fig. 5

Couverture de la revue Dyn - Dyn 4-5, Amerindian Number, décembre 1943 conçue par Walter Paalen. Coll. privée.
Le second texte paru dans le numéro spécial «Amerindian Number» (4-5) de Dyn se présente comme une étude ambitieuse sur le «Totem Art». L'objectif est d'attirer l'attention de ses contemporains sur la nécessité « d'intégrer », à la suite des arts africains et océaniens, «l'énorme trésor des formes amérindiennes dans la conscience de l'art moderne ${ }^{\mathbf{1 9}}$ ». Paalen est convaincu que grâce à une meilleure connaissance des cultures amérindiennes, il serait possible d'explorer des espaces mentaux inconnus transposables dans des formes abstraites, capables d'exprimer de profondes émotions (Alix 2000: 21 ; Pierre 2003). Les matériaux sur l'art de la côte Nord-Ouest qu'il tient pour être «parmi les plus grandes réalisations de tous les temps" nourrissent sa réflexion et lui donnent l'occasion de s'affirmer auprès de ses amis comme un éminent spécialiste de l'héritage artistique amérindien. Composé de quatre parties, le texte est richement illustré par les reproductions des objets acquis lors de son voyage dont il loue la rareté, à l'instar du paravent tlingit - figurant un ours grizzli - ou encore de deux masques de chamanes tlingit, mis en regard avec des pièces similaires collectées en Alaska lors de l'expédition Malaspina en 1792. Des photographies de Sulzer côtoient des gravures anciennes et des clichés de villages datant des années 1880, époque où les mâts héraldiques se dressaient fièrement devant les maisons des chefs 20. L'article a bénéficié des suggestions bibliographiques et des connaissances d'ethnologues tels George Emmons et William Newcombe avec lesquels il correspond: Emmons lui fournit des informations précieuses sur sa collection d'objets tandis que Newcombe le guide dans ses recherches bibliographiques et iconographiques (Winter 1992: 22 ; 2003: 160-163).

Paalen traite de l'art «totémique» dans une perspective historique négligeant d'étudier les caractéristiques du «fait totémique» tel qu'il se manifeste au sein de ces sociétés. Les matériaux ethnographiques spécifiques à la région sont essentiellement utilisés pour délimiter l'aire d'extension de l'art totémique et alimenter une discussion sur son ancienneté. Paalen fait abusivement remonter l'origine des «totems" au XVI" siècle, au prétexte que cet art au raffinement achevé serait le fruit d'un lent développement à l'égal de celui de l'art roman ou byzantin, la preuve étant qu'il était bien établi à l'arrivée des premiers explorateurs à la fin du XVIII' siècle. Pour Paalen, cet art indissociablement lié la mythologie est ancré dans une longue tradition - "les totems sont les colonnes vertébrales et des marqueurs de la vie sociale "-, à l'instar des arts d'Océanie et d'Asie qui présentent des affinités avec les arts des Amériques.

Pour comprendre l'art de la côte Nord-Ouest, dit-il, «nous devons entrer dans le monde du totem ", tâche difficile car les théories sur la question sont multiples et contradictoires (Paalen 1943: 18). En réalité, Paalen aborde la question sur un plan général en s'appuyant sur les théories évolutionnistes de John Ferguson McLennan et Wilhelm Wundt, faisant ainsi l'économie d'une analyse des formes d'organisation sociale telles qu'elles ont été décrites par Franz Boas dont il connaissait les travaux. Dans cette perspective, il défend l'idée que le totémisme "correspond à un stade de développement de la pensée archaïque dont on peut retrouver des traces dans toute l'humanité» et qui coïnciderait, à l'échelle humaine, au développement émotionnel d'un enfant par rapport à celui d'un adulte, argument soutenu par Sigmund Freud qui rapproche la vie psychique de l'enfant de celle de l'homme primitif. Comme les enfants, les «primitifs » n'ont aucun mal à s'identifier à un animal totémique car humains et non-humains sont dotés des mêmes facultés 
et de la même force vitale, de sorte qu'il n'existe aucune différence entre eux. Plus que l'absence de différenciation entre le subjectif et l'objectif, un des traits saillants de la mentalité « totémique » est l'identification sur le plan émotionnel de l'individu avec le monde environnant (ibid.): cette «identification affective» favorise, selon Paalen, la survenance d'un «climat magique» qui trouve à s'exprimer dans «les danses, les sacrifices, les repas cannibales, les orgies, les rites divinatoires et incantatoires ", c'est-à-dire dans l'action rituelle qui inclut une variété d'accessoires et d'objets conçus non pas comme des œuvres d'art mais comme des objets dotés d'une efficacité «car à ce stade magique, l'art est un véhicule et un moyen de l'action » (Paalen 1943: 20), remarque très pertinente de la part d'un artiste qui perçoit la capacité agentive de l'art. Dans son essai, Paalen veut mettre en évidence la dimension spirituelle et émotionnelle de l'art de la côte Nord-Ouest enracinée dans «les forces naturelles du cosmos ", telle qu'elle se manifeste dans ce qu'il définit comme le totémisme (Dickson 2014: 62; Rushing 1995: 121).

Dans ce texte, les matériaux ethnographiques posent le cadre culturel à partir duquel Paalen, en s'appuyant sur des théories anthropologiques auxquelles les non-spécialistes étaient encore sensibles, élabore une réflexion sur la nécessité d'inventer un langage pictural ${ }^{21}$ nouveau qui pourrait puiser son inspiration dans un modèle cosmologique où les choses, quelle que soit leur nature, sont connectées et en perpétuelle transformation. Loin de lui l'idée de se penser comme ethnographe dont la fonction, souligne-t-il, est "de remplir des herbiers indispensables à la connaissance, à faire le point du savoir », en un mot de faire œuvre d'archiviste de cultures en voie de disparition. II se place dans un autre ordre de réalité, celui de l'art: «À l'artiste philosophe de mettre au jour de la fatalité sensible [sic] de son époque, d'embrasser dans toute la latitude de la pensée et de l'espace ce qui se mue sans lui en trésor de poussières. " (Paalen 1942b: 48) L'art amérindien trouverait-il sa planche de salut dans son incorporation à l'art moderne qui à défaut d'en emprunter les caractéristiques formelles en conserverait l'esprit?

\section{Georges Duthuit: un éclairage sur le potlatch}

Grand connaisseur du fauvisme et de l'œuvre d'Henri Matisse dont il est le gendre, spécialiste de l'art copte et byzantin, Georges Duthuit est responsable, en 1929, de la rubrique «Art ancien» dans Cahiers d'art. Dans les années 1930, il noue des liens avec quelques surréalistes et s'intéresse aux arts dits «primitifs», lui-même à la recherche d'«une esthétique nouvelle» en rupture avec la tradition gréco-romaine de la représentation-imitation (Labrusse 2003: 66). À son arrivée à New York en 1941, il rejoint le cercle des écrivains et artistes surréalistes et, à partir de 1942, se retrouve annonceur dans l'émission «Voix de l'Amérique » produite par l'Office of War Information aux côtés de Breton et Lévi-Strauss. Il partage avec ses amis la même dilection pour les arts amérindiens et inuit d'Alaska, déjà éprouvée lors de ses visites dans la galerie de la côte Nord-Ouest de l'American Museum of Natural History et des autres musées d'ethnographie de la côte Est, mais dont il avait déjà pris la pleine mesure avec l'installation du mât héraldique de Seligmann au musée de l'Homme.

À l'instar de Breton, Lebel et Lévi-Strauss, Duthuit réunit une collection de masques d'Amérique du Nord auprès du célèbre antiquaire de la Troisième avenue, Julius Carlebach (Mauzé 2010 : 50-52), dont un impressionnant
21. Voir aussi «The New Image ", Paalen 1942a. 
22. Dans sa lettre à Picasso déjà citée, il écrit à propos de sa collection de masques: «II n'y en a pas d'autres en France, je crois, ou pas beaucoup et pas de cette taille. » (Labrusse 2010: 42) Son fils, Claude, évoque également l'achat d'un grand plat cérémoniel d'environ deux mètres de long figurant un chien (il s'agit d'un loup) (ibid. : 18-19). II est reproduit dans Labyrinthe.

23. Voir lettre de Duthuit à Breton du 14 décembre 1949, in DucheminPelletier 2008: 81, 90.

24. Le texte est repris en 1974 sous une forme légèrement modifiée.

25. Dans une lettre non datée (1949?) à Picasso, Duthuit réitère sa demande: «Il s'agissait d'un dessin ou de dessins à propos des Indiens de la Côte Nord-Ouest américaine (Matisse m'en a donné pour un livre que je prépare sur la Fête chez les Esquimos [...]. Pour les Indiens, j'ai un texte tout prêt et certains de mes amis me pressent de le publier plus convenablement que je ne l'ai fait- en partie - Labyrinthe [sic]. »

Une copie de la lettre m'a été remise en 2010 par Dominique Szymusiak.

\footnotetext{
ci-contre

fig. 6

Georges Duthuit,

"Le Don indien"

in Labyrinthe, $\mathrm{n}^{\circ} 18$

1er avril 1946, p. 11

Centre Pompidou,

Bibliothèque Kandinsky,

photo RMNGP.
}

masque à transformation kwakwaka'wakw et un autre yup'ik représentant un cygne et une baleine blanche formant une paire avec celui de Breton (Rousselot 2000: 436-438). Duthuit était un collectionneur déterminé dans ses choix tirant grande satisfaction de l'acquisition de pièces imposantes, tel le plat cérémoniel kwakwaka'wakw ${ }^{22}$. Dans une lettre à son époux en 1944, Isabelle Waldberg observe:

[Duthuit] [...] achète à tour de bras des objets primitifs ou autres [...] et [...] affirme son goût pour le grandiose ou comme il dit «l'impérial » en achetant des objets de très grandes dimensions tout en se demandant avec une certaine anxiété comment il les transportera en France. (Waldberg et Waldberg 1992: 276)

Duthuit témoignera du riche enseignement que lui ont apporté les arts et les cultures autochtones d'Amérique du Nord dans deux textes qui auraient dû conduire à la mise en place de projets éditoriaux plus ambitieux. «Le don indien " publié dans Labyrinthe, annonçait un second écrit à paraître, à l'initiative de Breton, dans un ouvrage intitulé Les Grands Arts primitifs d'Amérique du Nord, auquel devaient collaborer Lévi-Strauss et Lebel (Duchemin-Pelletier 2008: 80-81, 90). Il était aussi question d'un «livre sur les Esquimaux» pour lequel Duthuit avait demandé la participation de Breton et de Lebel ainsi que celle de Paul-Émile Victor pour la documentation (ibid. : 81) ${ }^{23}$. Mais Duthuit avait un autre projet en tête, celui d'une collection "consacrée aux rituels et au "sens de la fête" dans différentes civilisations" (Pulvénis de Séligny 2003: 47) où ethnographie et art moderne pourraient dialoguer.

Seul paraîtra en 1963 «Une fête en Cimmérie», long poème en prose dont la rédaction a commencé à New York en 1945, illustré par des dessins et des lithographies de Matisse représentant des portraits d' "Esquimaux » exécutés en 1949 (Szymusiak 2010). Ce texte évoque «une ballade imaginaire dans un New York devenu terre arctique » (C. Duthuit, 2003: 12), au cours de laquelle, s'identifiant à un chasseur yup'ik, il convoque les «esprits du silence et du secours au voyageur » qui se manifestent sous la forme de masques décrits avec la sensibilité du poète et la précision de l'ethnographe. En 1947, il passe commande «d'un ou de dessins" à Picasso, l'invitant à considérer les masques de la côte Nord-Ouest, qui présentaient, selon lui, des correspondances avec son œuvre:

Je voulais vous demander si ces masques vous intéressent, de venir les voir [...]. Pendant la longue insomnie new-yorkaise, ces affectueux démons ont été ma seule compagnie, ou à peu près, une espèce de protection nocturne, vraiment efficace. Sans trop savoir pourquoi, je les associais à vos images. Du fond lointain de la nature, ils me disaient des secrets [...]. Évidemment, les ethnographes, surtout de haut parage, sont un peu affolés du rapprochement et préféreraient voir le modernisme ailleurs que dans leur voisinage. Des liens vont apparaître au cours de cette confrontation. (Labrusse 2010: 42-43)

Picasso reste sourd à la demande de Duthuit qui s'en tient donc au seul article publié dans Labyrinthe en $1946^{\mathbf{2 4}}$ bien qu'il ait, semble-t-il, rédigé un texte beaucoup plus important sur l'art et les rituels des peuples de la côte Nord-Ouest ${ }^{25}$. 
№ 18

Jaอ̣pinatie
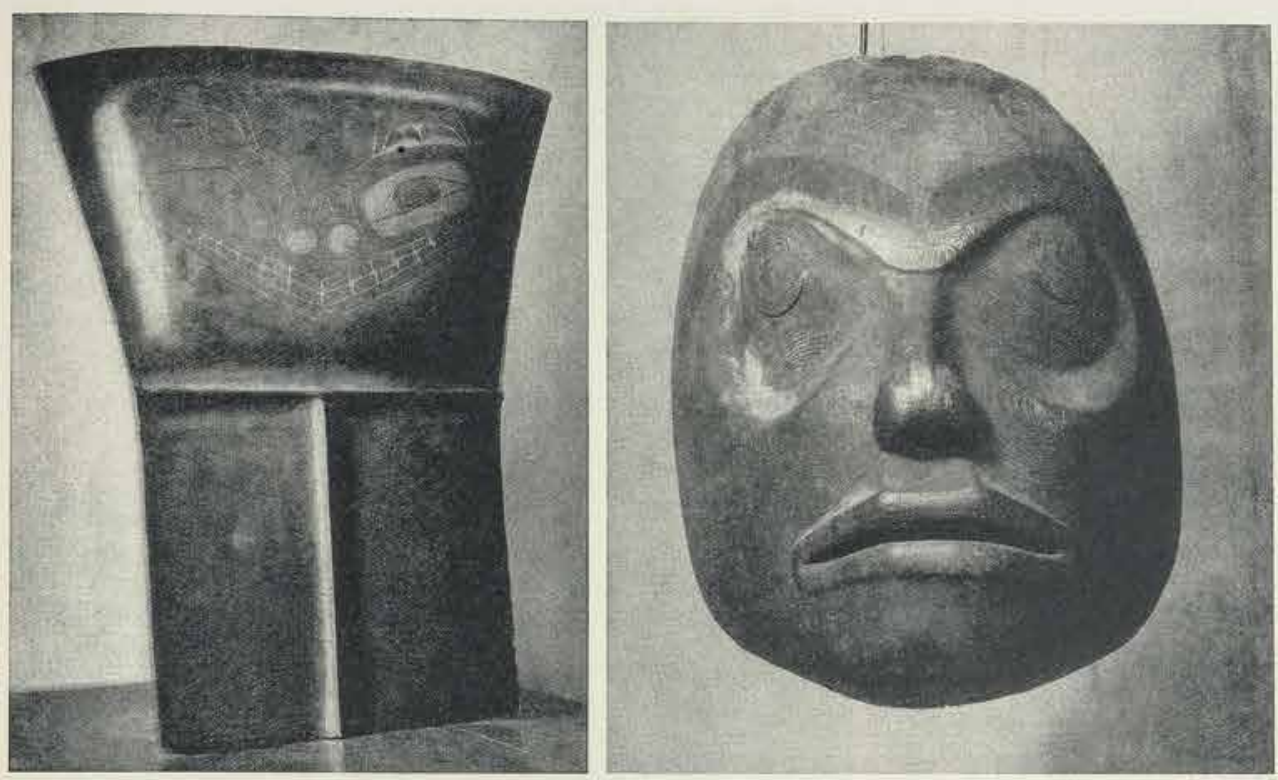

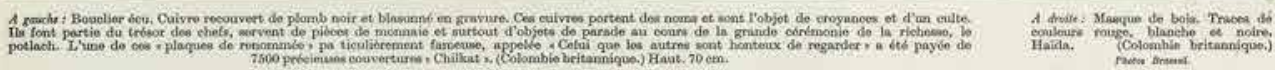

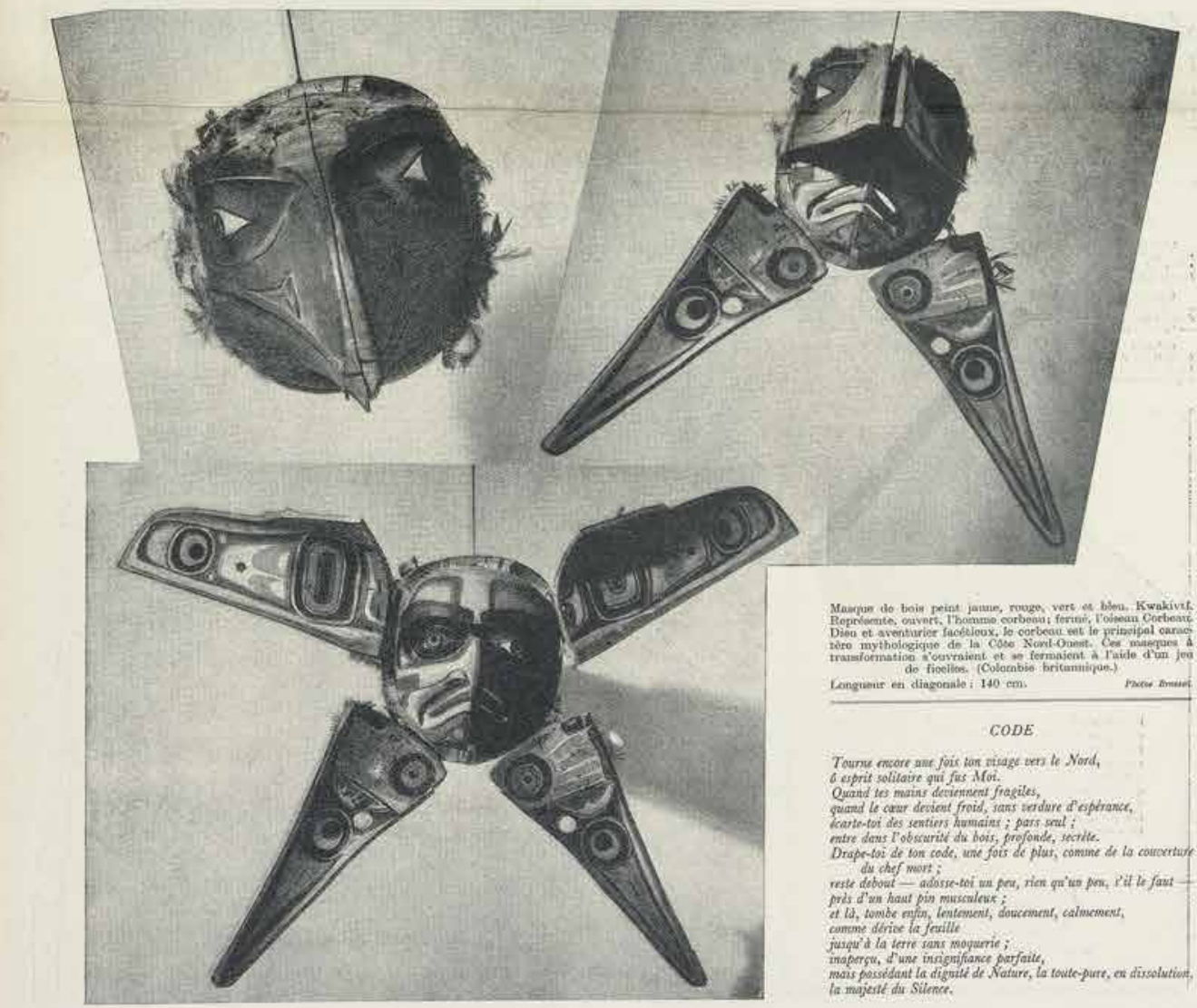


26. Les poèmes sont extraits de Songs of the Coast Dwellers (1930) de l'écrivaine et journaliste Constance Lindsay Skinner (1877-1939) qui, selon Duthuit, "s'inspire directement, surtout chez les Kiakut [sic] et les Haïdas, du geste et des sentiments de ses amis bruns les plus réfractaires à la civilisation, des spectacles d'une nature qui évidemment a fait une forte impression sur sa sensibilité de femme et de petite fille. "

(Duthuit 1946:10).

27. Cela indique que la rédaction de ce texte a commencé à New York.
«Le don indien sur la côte Nord-Ouest de l'Amérique, Colombie britannique» est d'un genre singulier mêlant écriture poétique, souci du détail ethnographique et analyse sociologique. II est nourri de données non référencées se rapportant essentiellement à l'ethnographie des Kwakwaka'wakw, aisément identifiables par les spécialistes. L'article est illustré dans le style des revues d'avant-garde des années trente par des photographies de Brassaï, des pièces de sa collection et un cliché de poteau de maison kwakwaka'wakw de Sulzer. II est enrichi de cinq courts poèmes, alternant avec les illustrations, faisant ainsi partie du dispositif iconographique ${ }^{26}$. À certains égards, ce texte manifeste une parenté avec l'article de Lévi-Strauss de 1943 dans l'évocation lyrique des êtres de la mythologie et des esprits qui prennent corps dans les œuvres d'art. II se rapproche des écrits de Paalen dans leur description suggestive des mâts dressés dans la solitude du crépuscule inspirant un sentiment de mélancolie, ces mâts étant les derniers témoins d'un monde révolu: "Nous parlons au passé», écrit Duthuit au tout début de son article (Duthuit 1974 [1946] : 315).

Dans un langage métaphorique, le critique décrit un univers où coexistent différents niveaux de réalité qui trouvent leur pleine expression dans l'art et les rituels. Un hommage est rendu aux mâts héraldiques, «hérissés de nageoires, de becs de rapaces, gonflés de narines carnassières et de paupières de batraciens, mêlant aux traits de l'homme ceux du corbeau, de la baleine-tueuse, de la lune ou du soleil [...]", qui donnent à voir des images complexes intégrant aux attributs physiques des personnages zoomorphes des éléments anthropomorphes, indices de l'intentionnalité humaine partagée par l'ensemble des entités qui composent le monde: «Les castors construisent des capitales, [...] la cuillère de corne bavarde avec le feu et la poutre de la maison, [...] les petites feuilles battent des mains " (ibid. : 315). À l'instar de Lévi-Strauss (1943; 1993: 173-175), Duthuit souligne la capacité des artistes à donner vie à un ensemble d'êtres participant de l'univers mythologique et surnaturel, et leur habileté à figurer la transformation, résultat d'une parfaite adéquation entre l'excellente maîtrise technique de l'artiste et la représentation d'une vision du monde. L'artiste, souligne-t-il, fait advenir «aux invisibles frontières du songe et du réel [...] la mutation de l'animal en homme et de I'homme en esprit: "les corps divins se manifestent par l'art" " (ibid. : 316). C'est l'occasion de rappeler la différence entre la conception de l'œuvre d'art classique en Occident où l'image se réduit à la simple représentation et celle existant sur la côte Nord-Ouest selon laquelle un objet devient une œuvre à la condition que celle-ci figure sous forme matérielle l'invisible et que l'image matérielle ainsi produite soit dotée de vie ou d'énergie. Comme les surréalistes, Duthuit retient que la valeur d'une œuvre dans les sociétés amérindiennes ne relève pas seulement du registre visuel ou esthétique mais a partie liée avec la performance rituelle, laquelle, tenant à la fois du théâtre et de la prestidigitation, crée les conditions de la croyance en la présence d'entités surnaturelles parmi les humains et lui donne ainsi sa véritable signification.

Après l'ample mémoration des rituels d'initiation qui doivent leur efficacité à l'illusionnisme - les exemples sont tirés des travaux de Boas (1897) -, il s'engage dans une discussion sur l'institution du potlatch en s'appuyant non pas sur des matériaux de première main mais sur les analyses de deux «ingénieux sociologues venus de l'autre côté de l'eau » (Duthuit 1974 [1946]: 318) ${ }^{27}$, à savoir «L'essai sur le don » de Mauss (1973 [1925]) et La Notion de 
dépense de Georges Bataille (1933), développée plus tard dans La Part maudite (1967 [1949]). L'auteur exprime sa fascination pour ces sociétés où l'on y «adore surtout la richesse et plus encore la dépense» (Duthuit 1974 [1946]: 315), reprenant ainsi les termes de Bataille ${ }^{28}$. Elles ont inventé une institution remarquable - le potlatch - définie comme «une sorte d'opéra cosmique qui tient du culte, du carnaval et du marché, du sacrifice et de la réception mondaine sublimée" (ibid. : 317) qui requiert le concours d'hommes nobles et riches, n'hésitant pas à détruire leurs biens pour écraser leurs rivaux. On sait aujourd'hui que cette manifestation est le résultat de la perversion du système de don et de contre-don dans un contexte colonial particulier marqué par l'introduction de nouveaux biens de prestige, la monétarisation des revenus et l'émergence d'une classe de nouveaux riches qui par la distribution ostentatoire de richesses essaya de se hisser au sommet de la hiérarchie sociale et d'occuper des positions laissées vacantes en raison du fort déclin de la population (Mauzé 1986). Duthuit reprend l'idée maîtresse de Mauss selon laquelle le potlatch n'est pas seulement un phénomène économique, mais un "fait social total » dans sa dimension à la fois religieuse, morphologique et esthétique, sollicitant l'ensemble de la société et de ses institutions dans le cadre de manifestations collectives. À la suite de Mauss et Bataille, il met l'accent sur les mécanismes de la dépense somptuaire (forme évoluée du don) dans le potlatch où la perte matérielle (voire la destruction de biens) se transforme en gain de prestige. "Dépense somptuaire et production utilitaire ne font qu'un » (Duthuit 1974 [1946] : 318), hypothèse qui interroge la pertinence des logiques économiques occidentales et met en évidence le contraste radical entre les sociétés de consumation chères à Bataille (les sociétés à potlatch) et les sociétés industrielles fondées sur le rationalisme, l'accumulation et le profit, face à un système extravagant qu'il décrit ainsi :

L'économie des habitants de la côte Nord du Pacifique ne souffre pas de ces délires de générosité. Elle ne cesse, au contraire de prospérer. À ces tournois du don indien, à ces fêtes du potlatch, s'offrent et se gaspillent des fortunes entières. [...] Le noble se dépouille de ses possessions les plus chères [...], en faveur d'un rival qu'il faut humilier, obliger à tout prix, ce qui lui donne d'ailleurs une chance de grandir à son tour [...]. Ces parieurs [...] peuvent briser des canots, brûler des villages, jeter à la mer [...] sous forme de plaques de cuivre, à la fois blasons et monnaie. (Ibid. : 317-318)

Bien qu'il ne soit pas de pure obédience surréaliste, on retrouve chez Duthuit la même exaltation que chez certains de ses compagnons, celle de traits censés caractériser les peuples non-occidentaux qui sont autant de contre-exemples ou d'inversions des pratiques et valeurs propres à la civilisation occidentale. L'auteur célèbre ici des cultures qui accordent une grande place au sens du théâtre et de la fête, au prestige et à l'ostentation, où l'art est indissociable de la vision du monde, en radicale opposition avec ce que peut offrir la société occidentale dans ces temps particulièrement troublés de la Seconde Guerre mondiale, par deux fois évoqués dans le texte.
28. Duthuit avait participé aux séances du Collège de sociologie entre 1937 et 1939 (Hollier 1979).

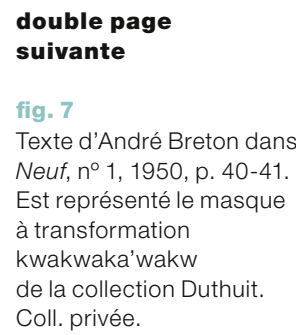




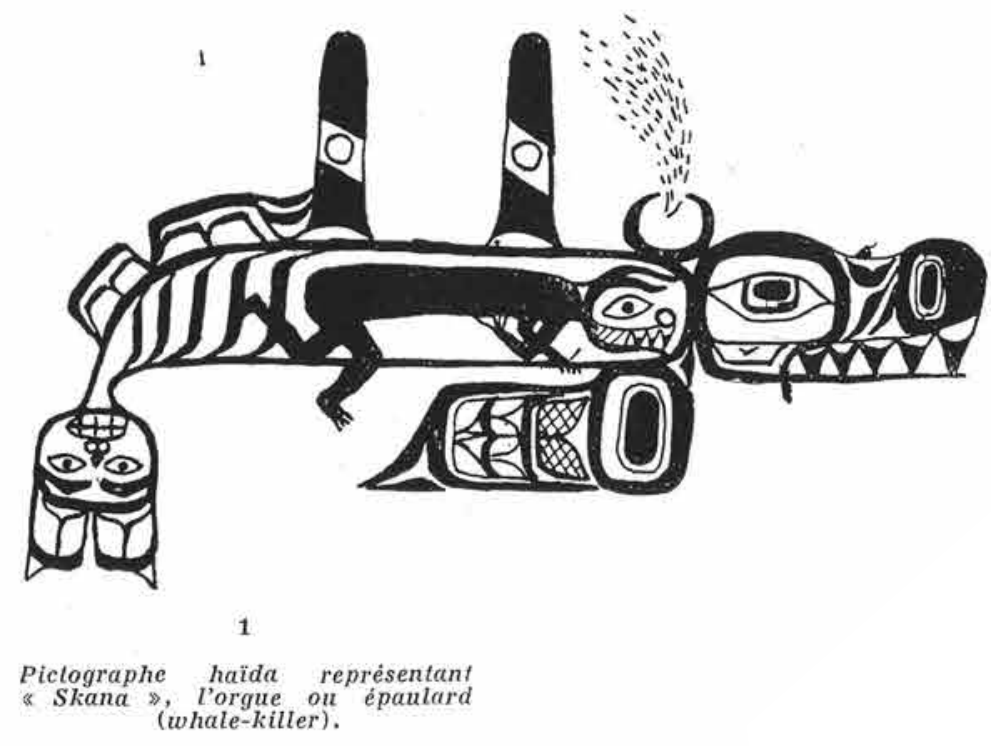

2

Masque représentant Toiseav. tonnerre.

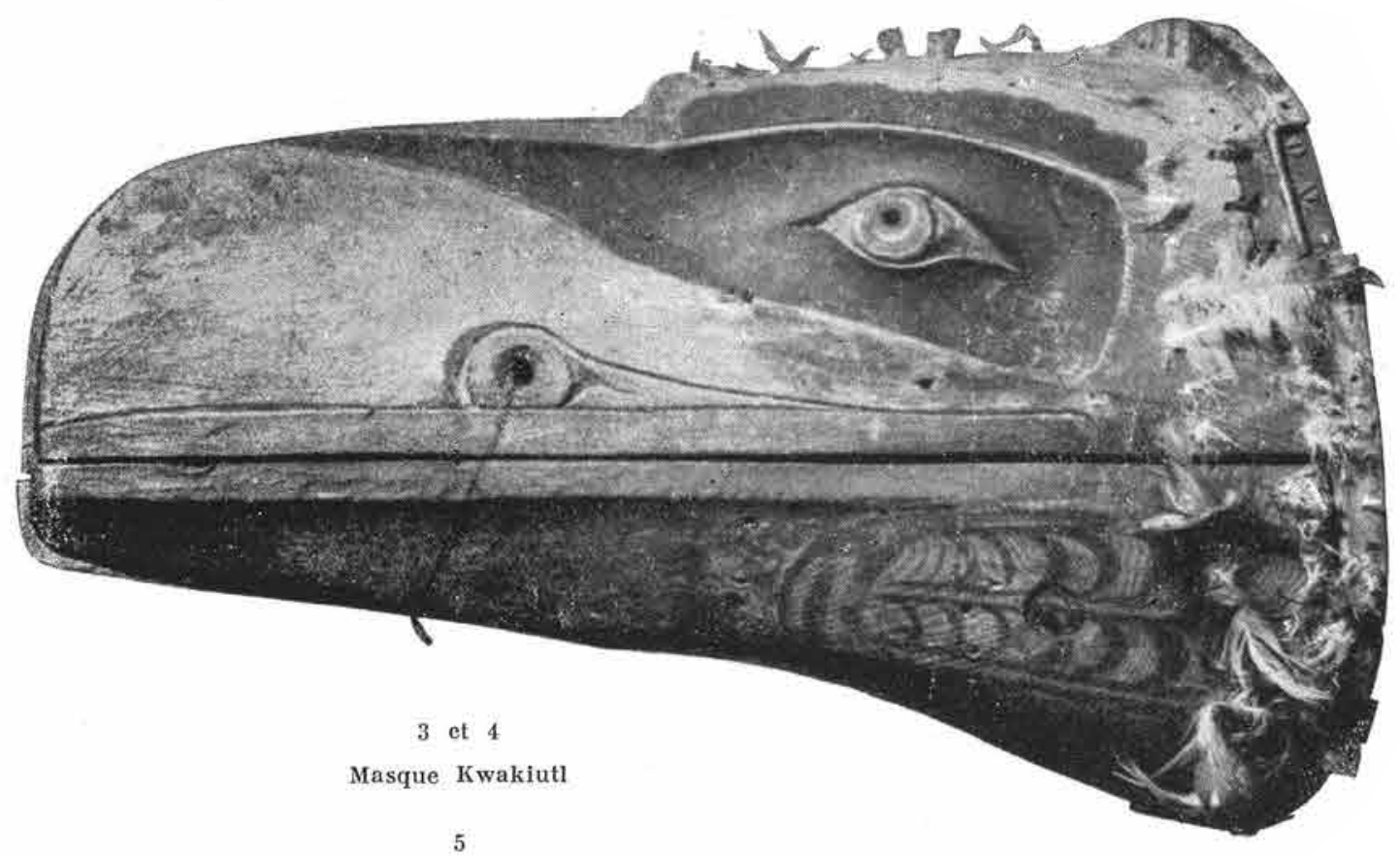

Masque figurant ti serpent $\dot{\alpha}$ deux tètes. Le visage central représente letes. Le visage central represent avec ses deux plumes. A chaque avec ses deux plumes. A chaque
cxtrémité se trouvent des têtes de cxtrémité se trouvent des têtes de serpent a plume qui peuvent, au rentrées. peuvent être pliés en avant et en arrière. - Utilisé dans les danses d'hiver comme représentation pan tomimique de la légende du vison. 

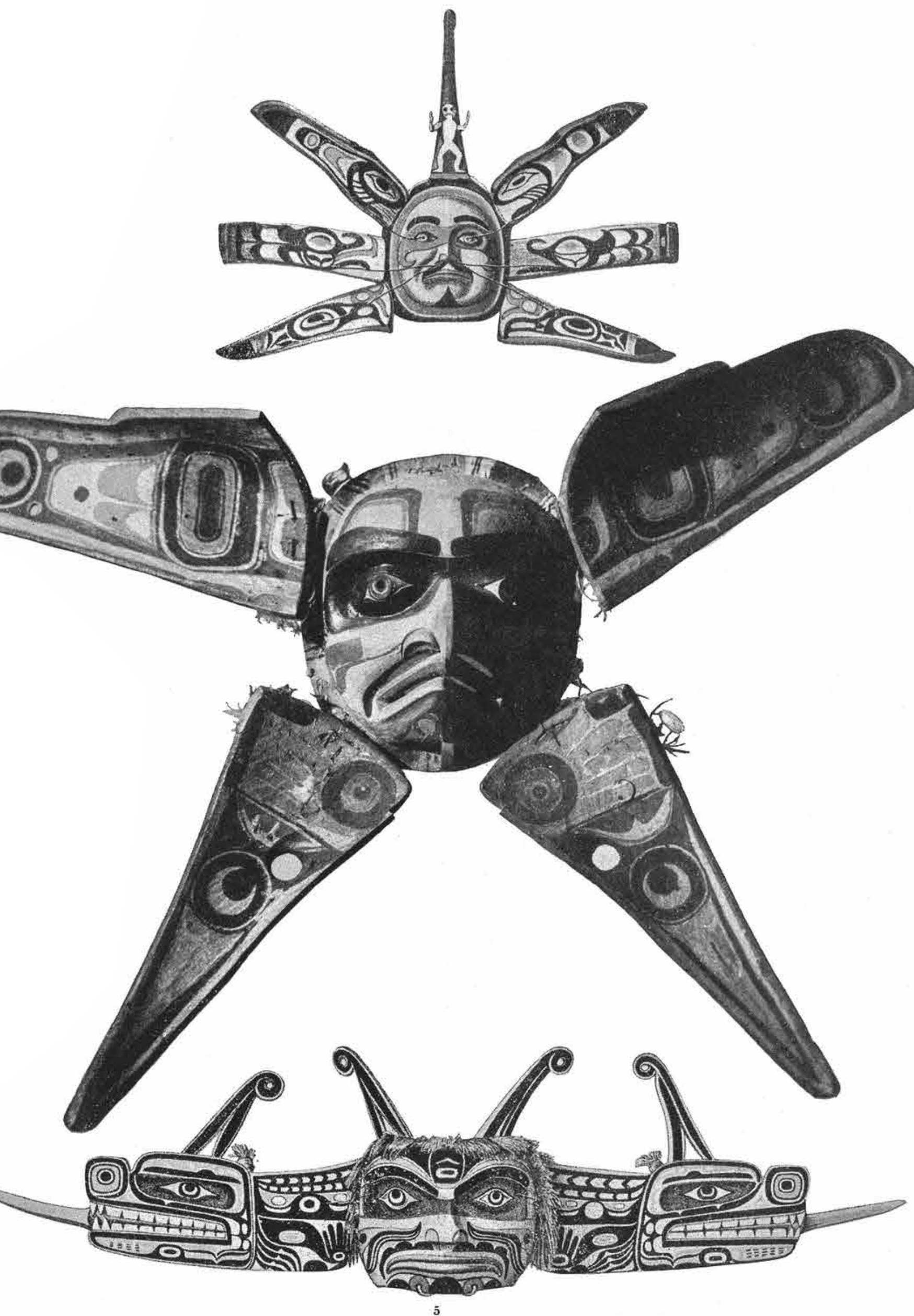


\section{ci-contre}

fig. 8

Couverture de la revue Neuf $n^{\circ} 1$, juin 1950.

L'illustration est un masque haisla de l'ancienne collection Breton.

Coll. privée.
L'Amérique du Nord-Ouest de Duthuit appartient à deux registres de temporalité - le passé et le présent: un passé atemporel et un présent qui inclut le moment historique du contact en passant par la période coloniale. Le changement de temporalité n'est pas marqué par un changement de temps grammatical mais est indiqué par l'usage constant du présent. Le recours à ce procédé littéraire conduit à figer les cultures dans une sorte d'atemporalité imaginaire tout en les inscrivant paradoxalement dans un présent historique qui leur confère une contemporanéité au moment où elles vont disparaître.

\section{André Breton: la fascination pour le masque}

Bien qu'André Breton ait évoqué à maintes reprises son attirance pour l'art de la côte Nord-Ouest dans ses écrits, poèmes et entretiens, «Note sur les masques à transformation de la côte du Pacifique Nord-Ouest » (1950) est le seul texte significatif sur l'art de cette région publié sous sa signature. II paraît dans le premier numéro de Neuf, revue de la Maison de la médecine qui, dans le sillage des revues d'avant-garde (Documents et Minotaure) des années 1930, accueillait des textes sur l'art et l'ethnographie, et accordait une grande place à la photographie (Perrigault 2010).

Le texte de Breton s'ouvre sur un poème intitulé "Chant du chef, poème kwakiutl» et comprend plusieurs illustrations dont la mise en page n'est pas sans rappeler celle inaugurée par Paalen dans "Totem art». On y reconnaît des photographies de masques à transformation publiées dans The Kwakiutl of Vancouver Island (1909) de Boas (Bonnet 1985: 23; Mauzé 1992: 295) et celle du masque de Duthuit par Brassaï, parue dans Labyrinthe. Les deux masques articulés de Breton font aussi partie du dispositif iconographique: le masque polychrome haisla illustre la couverture; le masque articulé haida s'impose en première page de l'article.

Breton introduit son propos en reprenant à son compte l'idée de Georges Buraud (1948) selon laquelle le masque est un lieu d'échanges entre "les forces occultes qui mènent le monde " et les forces de l'inconscient dominées par les émotions et les instincts - conception renvoyant au fonctionnement mental des sociétés dites archaïques, déjà largement remis en cause à cette époque. D'une manière plus générale, Breton s'interroge dans ce texte sur la capacité du masque à combler le désir de tout humain - indépendamment de sa tradition culturelle de «devenir autre que ce qu'il est» (Suzuki 1996: 53), réflexion approfondie dans "Le Phénix du masque" (1959) où il affirme: «Nous pouvons mesurer [...] l'étendue des prestiges qui s'attache à la transfiguration, aussi bien qu'à l'éclipse, de ce que représente d'individuel l'aspect du visage humain. » (Breton 1970a [1959] : 185)

En introduction, il pose les conditions de sa relation aux objets en opposition avec celles des ethnologues et des muséographes. Breton, on le sait, critique sévèrement la prétention scientifique de ces derniers, les accusant, dans un cadre muséal, de détourner les objets de leur contexte d'usage et de les dépouiller de leur sacralité. II plaide pour une approche sensible de l'objet, l'émotion permettant seule d'en saisir la "vérité» en dehors de toute considération scientifique, point de vue qui n'était pas partagé par Marx Ernst qui, selon Lévi-Strauss, était curieux d'ethnologie et «voulait [...] tout savoir " sur les objets qu'il collectionnait (Lévi-Strauss et Éribon, 1988: 53). Dans un autre registre, celui de l'appréciation esthétique, il dénonce la démarche des 


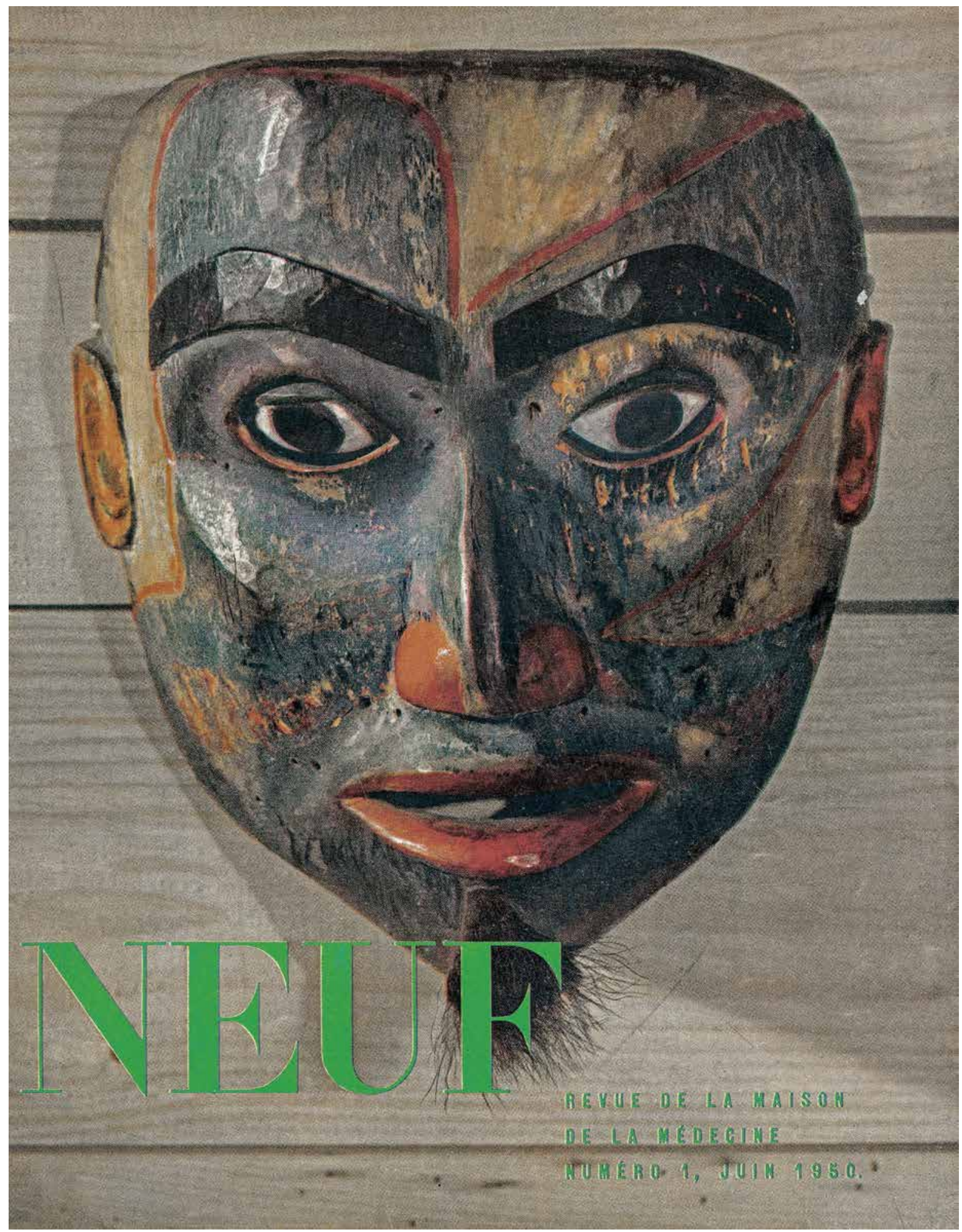




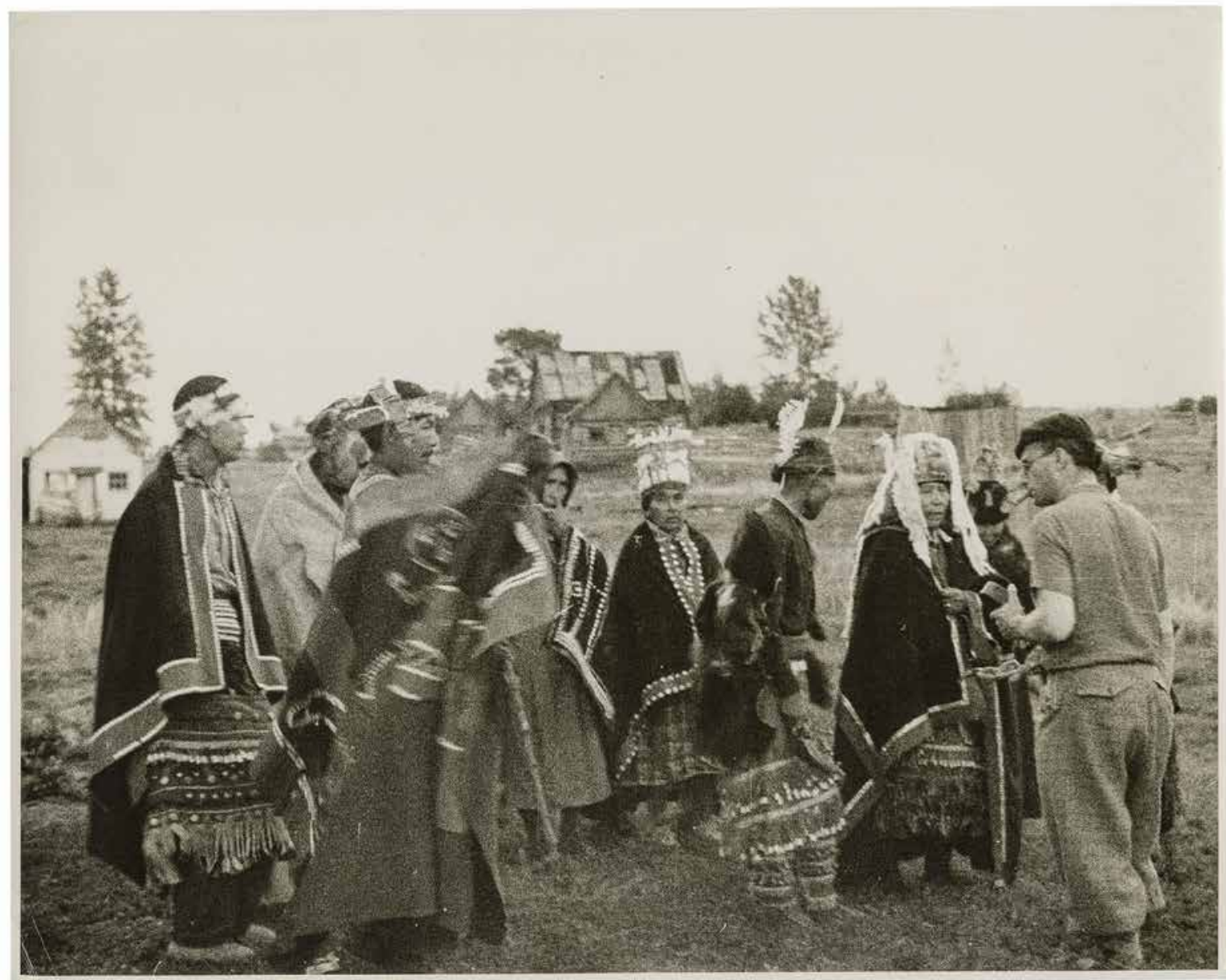

fig. 9

Arlette Seligmann,

Kurt Seligmann durant sa

visite dans les territoires

du Nord-Ouest, à Hagwilget,

avec un groupe de

danseurs wetsuwet'en

revêtus de leurs costumes

cérémoniels, 1938-1939.

Musée canadien de

I'histoire, IMG2016-0074-

0022-Dm. 
collectionneurs qui sélectionnent les œuvres en fonction de critères (ancienneté, patine, réussite plastique, valeur commerciale, etc.) qui, comme les «savantes gloses ", sont autant d'obstacles à l'imagination qui en révèle la force poétique. Breton a toujours revendiqué la priorité de l'approche sensible sur le savoir: «Aimer, d'abord, il sera toujours temps ensuite de s'interroger sur ce qu'on aime jusqu'à n'en plus vouloir rien ignorer. » (Breton 1970a [1959]: 221-222)

Dans son texte, il cite longuement l'article de Lévi-Strauss (1944-1945) sur le dédoublement de la représentation dans l'art de la côte Nord-Ouest, recherche présentée à l'École libre des hautes études en 1942 (Waldberg et Waldberg 1992: 184). À la différence de Duthuit, il ne s'aventure pas dans une description savante du potlatch qu'il laisse aux spécialistes, bien qu'il en dégage quelques traits pertinents au détour d'une seule phrase, montrant qu'il est au courant des débats sur la complexité des institutions sociales chez les Kwakwaka'wakw (Breton 1950: 41). Breton traite du potlatch sur un mode qui lui est familier - celui de la poésie - en juxtaposant à son article un «Chant du chef Poème kwakiutl », ce qui lui permet de restituer à sa manière l'atmosphère du rituel. Par cet artifice, il nous conduit au cœur d'une cérémonie à l'occasion de laquelle le chef yaqatlenlis convoque un grand nombre d'invités. Le poème se présente sous la forme d'un discours prononcé par le chef avant la distribution des cadeaux, où celui-ci fait valoir son rang et son extrême richesse contre laquelle nul ne peut rivaliser sous peine de subir défaite et humiliation.

CEuvre de Breton, ce poème est un collage incluant des extraits de discours de chefs prononcés lors de l'achat d'un cuivre et de chants recueillis par Boas, traduits en français (Mauzé 1992: 293, 296). De manière convaincante, Breton réorganise ces fragments disparates dans le respect de la rhétorique cérémonielle kwakwaka'wakw conservant ainsi la saveur du langage métaphorique au moyen duquel est exaltée la magnificence des chefs de noble extraction. Certes, Breton s'exprime moins en ethnographe qu'en poète, cependant malgré ses déclarations intempestives contre le savoir ethnographique, l'exercice met en évidence l'utilisation des matériaux comme embrayeur d'une inspiration littéraire.

Les surréalistes ont accordé une valeur poétique au mythe moins pour ses qualités formelles que parce qu'ils le considéraient comme une forme d'expression de l'inconscient témoignant d'une extraordinaire faculté d'imagination. En réalité, ils étaient attentifs à toutes les formes d'expression orale de la parole indigène, à l'exemple de Péret, qui dès son arrivée au Mexique, s'attèle à explorer les arts poétiques des sociétés amérindiennes dans son Anthologie des mythes, légendes et contes populaires d'Amérique (Péret 1960). Si la pensée mythique a pu être une source d'inspiration pour Breton, dans son «poème kwakiutl», il met à l'honneur l'importance de l'art oratoire des Kwakwaka'wakw qui prend sa pleine signification dans le rituel.

Dans son étude, Breton insiste sur deux points: les qualités plastiques des masques à transformation et leur force de suggestion. Composés de plusieurs volets mobiles, les masques sont manipulés par le porteur de telle sorte qu'un masque peut s'ouvrir en plusieurs parties, dévoilant ainsi d'autres masques représentant les différentes apparences physiques d'un ancêtre ou d'un esprit, ou rendant compte des états émotionnels de l'entité 
figurée. Pour l'auteur, ce type d'objet alliant invention plastique et matérialisation d'un désir profond de devenir autre force d'autant plus l'admiration que la transformation, grâce au mécanisme, opère un effet de surprise:

La vertu [des masques à transformation] réside avant tout dans une possibilité de passage brusque d'une apparence à une autre, d'une signification à une autre. II n'est pas une œuvre statique, si réputée soit-elle, qui avec celle-ci, puisse supporter la comparaison sous le rapport de la vie (et de l'angoisse). (Breton 1950: 39)

C'est dans la théâtralité du rituel que les masques à transformation dévoilent leur pleine signification. Nul doute que Breton partage l'idée de Lévi-Strauss que le rôle des masques "est d'offrir une série de formes intermédiaires, qui assurent le passage du symbole à la signification, du magique au normal, du surnaturel au social " (Lévi-Strauss 1958: 289). À quoi il ajoute que leur qualité émotionnelle tient au fait que la transformation évoquée dans le mythe est donnée à voir de manière spectaculaire dans le rituel:

Dans le raccourci lyrique d'une séance d'initiation - du poisson à l'oiseau, de l'oiseau à l'homme -, ils embrassent un des plus grands vertiges humains en réalisant le transformisme non seulement en pensée mais en action. (Breton 1950: 41)

Pour Breton, ces masques nous interpellent au-delà de leur contexte singulier de signification. Plus que n'importe quelle œuvre "sauvage", le masque à transformation répondait à ce désir de "déroutement de l'esprit que réclamaient les surréalistes" (Waldberg 1965: 20) dans sa capacité à brouiller les frontières entre le réel et l'imaginaire et à faire surgir le merveilleux par surprise.

Concluons sur le constat que nombre des textes présentés ont été conçus à un moment où l'expertise des surréalistes en matière d'art primitif était reconnue (Leclerc 2010: 367-370) et qu'à ce titre ils affirmaient leur légitimité en tant qu'auteurs. Notons qu'ils reposent sur une bonne connaissance des travaux sur les sociétés de la côte Nord-Ouest - notamment ceux de Boas, de Barbeau et du jeune Lévi-Strauss de la période new-yorkaise bien qu'ils s'inscrivent d'une manière générale dans un cadre théorique déjà largement contesté à l'époque dans la compréhension de la pensée "primitive».

L'ethnologie n'est pas toujours bien traitée par les surréalistes qui en ont essentiellement prélevé des fragments déconnectés de la réalité ethnographique pour décaler leur regard sur la civilisation occidentale et nourrir leur imaginaire créatif. «Le Surréalisme a eu besoin de l'ethnologie, mais l'inverse n'est pas vrai » (Blachère 1996: 129); toutefois, encore, comme le montre cette étude, faudrait-il établir des distinctions dans le rapport que chacun des membres du mouvement surréaliste entretient avec l'ethnographie. Les ethnologues ont critiqué l'amateurisme des surréalistes, leur traitement fantaisiste, poétique et non scientifique des faits ethnographiques et leur emploi approximatif de notions précises. Cependant, on note que Seligmann a produit un texte digne d'être publié dans une revue d'ethnologie et que Paalen portait un intérêt au détail ethnographique pour nourrir ses hypothèses. De même, on ne peut manquer de souligner que certains d'entre eux ont 
exploré - certes dans leur propre perspective - des champs d'investigation qui n'avaient pas encore été abordés par l'ethnologie. II serait certainement abusif d'affirmer qu'ils sont les précurseurs, dans un nouveau domaine au carrefour de la littérature et de l'ethnologie, de l'ethnopoétique apparue dans le champ anthropologique dans les années soixante-dix, mais on doit porter à leur crédit d'avoir manifesté un intérêt pour la littérature orale autochtone et de lui avoir attribué un statut équivalent à celui de la littérature occidentale. En accordant une place centrale au discours d'un chef kwakwaka'wakw, Breton reconnaît les qualités poétiques de la parole rituelle et sa fonction performative dans certaines circonstances d'énonciation, le type d'interrogation qui aujourd'hui nourrit les travaux sur l'oralité. Dans le domaine de l'art, l'approche des surréalistes fondée sur l'émotion esthétique comme mode exclusif de connaissance a pu être critiquée, mais il apparaît que la conception de Duthuit ou de Breton se rapproche de celle de Lévi-Strauss pour qui croyance au surnaturel et émotion esthétique ont partie liée, la première fonde la seconde qui, à son tour, la valide.

Laboratoire d'anthropologie sociale, Collège de France, EHESS, CNRS

PSL Research University Paris France marie.mauze@college-de-france.fr 


\section{Adam, Leonhard}

1923 Nordwest-Amerikanische Indianerkunst. Berlin, Wasmuth.

1934 «Sculptures en bois de l'Amérique du Nord-Ouest ", Cahiers d'art $9(1-4): 59-65$

\section{Alix, Josephina}

2000 « L'art indigène et la nature. L'Ancien et le Nouveau Monde: rencontre de deux arts ", in Carlos Ortega et Stéphanie Molinard (dir.), Les Surréalistes en exil et les débuts de l'école de New York, catalogue d'exposition (Madrid, Museo Nacional Centro de Arte Reina Sofía et Strasbourg, musée d'Art moderne et contemporain). Strasbourg, Musées de Strasbourg: 17-37.

\section{Barbeau, Marius}

1929 Totem Poles of the Gitksan: Upper Skeena River, British Columbia. Ottawa, F. A. Acland.

s. d. "Les mémoires de Marius Barbeau ", fonds Carmen Roy, archives du Musée canadien de l'histoire, Gatineau (Québec, Canada).

\section{Bataille, Georges}

1967 [1949] La Part maudite, précédé de La Notion de dépense, préface de Jean Piel. Paris, Les Éditions de minuit.

\section{Bédouin, Jean-Louis}

1961 Vingt ans de surréalisme: 1939-1959. Paris, Denoël.

\section{Blachère, Jean-Claude}

1996 Les Totems d'André Breton: surréalisme et primitivisme littéraire. Paris, L'Harmattan.

\section{Boas, Franz}

1897 «The Social Organization and the Secret Societies of the Kwakiutl Indians ", Report of the United States National Museum for 1895.

1909 The Kwakiutl of Vancouver Island, The Jesup North Pacific Expedition, Memoirs of the American Museum of Natural History, vol. V, part II. Leiden, E. J. Brill.

\section{Bonnet, Marguerite}

1985 "À partir de ces "mécaniques à la fois naïves et véhémentes... " ", Pleine Marge 1 : 18-28.

\section{Breton, André}

1937 L'Amour fou. Paris, Gallimard.

1950 "Note sur les masques à transformation de la côte pacifique Nord-Ouest ", Neuf 1: 36-41, repris dans Pleine Marge $1:$ 9-15.

1970a [1959] "Le Phénix du masque ", in Perspective cavalière. Paris, Gallimard: 182-186.

1970b "Main première », in Perspective cavalière. Paris, Gallimard: 221-225.

2003 André Breton, 42 rue Fontaine. Tome VIII : Arts primitifs. Catalogue de la vente publique de la collection André Breton. Paris, CalmelsCohen.

\section{Buraud, Georges}

1948 Les Masques. Paris, Le Seuil.

\section{Covarrubias Miguel et Rubín de la Borbolla, Daniel (dir.)}

$1945 \mathrm{El}$ arte indígena de Norteamérica, exposition au Museo nacional de Antropología, du 20 mars au 20 avril 1945, Mexico, Insituto Nacional de Antropología e historia.

\section{Cowling, Elizabeth}

1978 "The Eskimos, the American Indians and the Surrealists ", Art History 1 (4) : 484-500.

\section{Dawn, Leslie}

2006 National Visions, National Blindness: Canadian Art and Identities in the 1920s. Vancouver, UBC Press.

\section{Dickson, Kent}

2014 "Surrealist Views, American Landscapes: Notes on Wolfgang Paalen's Ruin Gazing ", Journal of Surrealism and the Americas 8 (1) 51-73.

\section{Duchemin-Pelletier, Florence}

2008 "Surréalisme et art inuit », Journal of Surrealism and the Americas 2 (1) : 64-94.

\section{Duthuit, Claude}

2003 "Une fête en Cimmérie ", in Claude Duthuit, Isabelle Monod-Fontaine, Claudine Grammont, Marie-Thérèse Pulvénis de Séligny (dir.), Une fête en Cimmérie: représentation du visage dans l'œurre de Matisse, catalogue d'exposition (Nice, musée Matisse). Paris, RMN : 7-13.

2010 "Esquimaux et arts derniers", in Dominique Szymusiak (dir.), Les Esquimaux vus par Matisse: Georges Duthuit, une fête en Cimmérie, catalogue d'exposition (Le Cateau-Cambrésis, musée départemental Matisse).

Paris, Hazan: 11-23.

\section{Duthuit, Georges}

1946 «Le don indien sur la côte Nord-Ouest de l'Amérique, Colombie britannique",

Labyrinthe 18: 9-11, repris dans Représentation et présence premiers écrits et travaux 1974. Paris, Flammarion: 315-319.

\section{Hauser, Stefan}

1997 Kurt Seligmann, 1900-1962 Leben und Werk. Bâle, Schwabe Verlag.

\section{Hollier, Denis}

1979 Le Collège de sociologie (1937-1939). Paris, Gallimard.

\section{Kloyber, Christian (dir.)}

2000 Wolfgang Paalen's Dyn: The Complete Reprint. Vienne/New York, Springer.

\section{Labrusse, Rémi}

2003 "Le muséoclaste", in Rémi Labrusse et al. Autour de Georges Duthuit, catalogue d'exposition (Aix-en-Provence, Galerie d'art du conseil général des Bouches-duRhône). Arles, Actes Sud: 57-86.

2010 "Chimérique Cimmérie, Georges Duthuit et les arts amérindiens ", in Dominique Szymusiak (dir.), Les Esquimaux vus par Matisse: Georges Duthuit, une fête en Cimmérie, catalogue d'exposition (Le Cateau-Cambrésis, musée départemental Matisse). Paris, Hazan : 25-46.

\section{Leclerc, Sophie}

2010 La Rançon du colonialisme: les surréalistes face aux mythes de la France coloniale (1919-1962). Dijon, Les Presses du réel.

\section{Lévi-Strauss, Claude}

1943 «The Art of the Northwest Coast at the American Museum of Natural History", Gazette des beaux-arts : 175-182, repris in La Voie des masques. Paris, Plon, 1979.

1958 "Le dédoublement de la représentation dans les arts de l'Asie et de l'Amérique ", in Anthropologie structurale. Paris, Plon: 269-299.

1993 Regarder Écouter Lire. Paris, Plon.

\section{Lévi-Strauss, Claude et Éribon, Didier}

1988 De près et de loin. Paris, Odile Jacob.

\section{Maurer, Evan}

1991 "Dada et surréalisme », in William Rubin (dir.), Le Primitivisme dans l'art $d u x x^{e}$ siècle. Paris, Flammarion: 535-594.

\section{Mauss, Marcel}

1973 [1925] «Essai sur le don, forme et raison de l'échange dans les sociétés archaïques», in Sociologie et Anthropologie. Paris, PUF.

\section{Mauzé, Marie}

1986 «Boas, les Kwagul et le potlatch: éléments pour une réévaluation ", L'Homme 100: 21-63.

1992 «Premiers contacts entre les surréalistes et l'art de la côte Nord-Ouest: le tambour d'eau des castors ", in Joëlle Rostkowski et Sylvie Devers (dir.), Destins croisés : cinq siècles de rencontres avec les Amérindiens. Paris, Albin Michel/Unesco : 283-296.

2008 "Totemic Landscapes and Vanishing Cultures through the Eyes of Wolfgang Paalen and Kurt Seligmann ", Journal of Surrealism and the Americas 2 (1): 1-21.

2010 "Les esprits du silence», in Dominique Szymusiak (dir.), Les Esquimaux vus par Matisse: Georges Duthuit, une fête en Cimmérie, catalogue d'exposition (Le Cateau-Cambrésis, musée départemental Matisse). Paris, Hazan: 47-57. 


\section{Meunier, Yannick}

2008 "Kurt Seligmann et Marius

Barbeau, chronique d'une rencontre décisive ", in Lynda Jessup, Andrew Nurse et Gordon Ernest Smith (dir.), Around and About Marius Barbeau: Modelling Twentieth-Century Culture. Gatineau (Québec), Musée canadien des civilisations: 79-94.

\section{Métraux, Alfred et Bing, Fernande}

1964 "Entretiens avec Alfred Métraux", L'Homme 4 (2) : 20-32.

\section{Neufert, Andreas}

2011 "Ten rolls of film documenting Wolfgang Paalen's Journey through British Columbia in Summer 1939 ", in Dawn Ades (dir.), The Colour of my Dreams. Vancouver, Vancouver Art Gallery: 229-235.

\section{Paalen, Wolfgang}

1942a "The New Image", Dyn 1 : 10-15.

1942b "Paysage totémique " Dyn 1: 46-50.

1942c "Paysage totémique», Dyn 2: 42-47.

1942d "Paysage totémique», Dyn 3: 27-31.

1943 «Totem Art ", Dyn 4-5 : 7-37.

1994a "Paysage totémique", dont les trois parties sont reprises in Pleine Marge 20: 35-50.

1994b "Voyage Nord-Ouest ", Pleine Marge 20 : 11-33.

\section{Péret, Benjamin}

1939 «Clou du Musée de l'Homme et inauguré hier soir. Krikiett, le plus haut totem d'Europe [...]", Paris-Soir, 21 janvier 1939.

1960 Anthologie des mythes, légendes et contes populaires d'Amérique. Paris, Albin Michel.

\section{Perrigault, Laurence}

2010 "La surprenante imagerie médicale de la revue Neuf ", Strenæ [en ligne], disponible sur: http:// strenaerevuesorg/73 (consulté le 14 mars 2016)

\section{Pierre, José}

2003 "Wolfgang Paalen au

carrefour de la "primitivité" et de la modernité ", Art tribal 4 : 56-65.

\section{Pulvénis de Séligny, Marie-Thérèse}

2003 «Une fête en Cimmérie - de l'autoportrait au masque", in Claude Duthuit et al. (dir.), Une fête en Cimmérie: représentation du visage dans l'œuvre de Matisse, catalogue d'exposition (Nice, musée Matisse). Paris, RMN

\section{Rousselot, Jean-Loup}

2000 "Sculpture yu'pik (inuit) ", in Jacques Kerchache (dir.),

Sculptures. Paris, RMN : 436-438.

\section{Rubin, William}

1991 "Introduction ", in William Rubin (dir.), Le Primitivisme dans l'art moderne. Paris, Flammarion: 1-81.

\section{Rushing, Jackson}

1995 Native American Art and the New York Avant-Garde: a History of cultural Primitivism. Austin, University of Texas Press.

\section{Sawin, Martica}

1995 Surrealism in Exile and the Beginning of the New York School. Cambridge (États-Unis)/Londres, The MIT Press.

\section{Seligmann, Kurt}

1938 "Imageries cinghalaises",

Revue du xx siècle 2: 22.

1939a "Keigyett: Myth of the Totem of Gyadem Skanees", Revue du $x x^{e}$ siècle 5-6: 28

1939b "Le mât-totem de Gédem skanisk (Gyaedem Skanees) ", Journal de la Société des Américanistes 31: 121-128.

1939c «Entretien avec un Tsimshian ", Minotaure 12-13: 66-68.

s. d. Manuscript describing the location of the Gitksan, the circumstances of the purchase of the pole, fonds Kurt Seligmann, VII-C-103M, boîte 306f1, Gatineau,

Canada, Musée canadien de l'histoire.

\section{Szymusiak, Dominique}

2010 «Matisse illustre le texte de Georges Duthuit, "Une fête en Cimmérie" ", in Dominique Szymusiak (dir.), Les Esquimaux vus par Matisse: Georges Duthuit, une fête en Cimmérie, catalogue d'exposition (Le Cateau-Cambrésis, musée départemental Matisse). Paris, Hazan: 71-104.

\section{Suzuki, Masao}

1996 «L'entrée des masques: André Breton et le mythe comme événement ", Pleine Marge 7 : 49-60.

\section{Waldberg, Patrick}

1965 Chemins du surréalisme. Bruxelles, Éditions de la Connaissance.

\section{Waldberg, Patrick et Waldberg, Isabelle}

1992 Michel Waldberg (dir.), Un amour acéphale:

correspondance 1940-1949.

Paris, Éditions de la Différence.

\section{Winter, Amy}

1992 «The Germanic Reception of Native American Art: Wolfgang Paalen as Collector, Scholar, and Artist", European Review of Native American Studies 6 (1) : 17-26.

2003 Wolfgang Paalen: Artist and Theorist of the Avant-Garde. Westport (États-Unis), Praeger. page 180 et ci-contre Kurt Seligmann, Croquis de la frontière entre les territoires gitksan et carrier, 1938 (détail). Musée canadien de l'histoire, IMG2008-0913-0001-Dm.
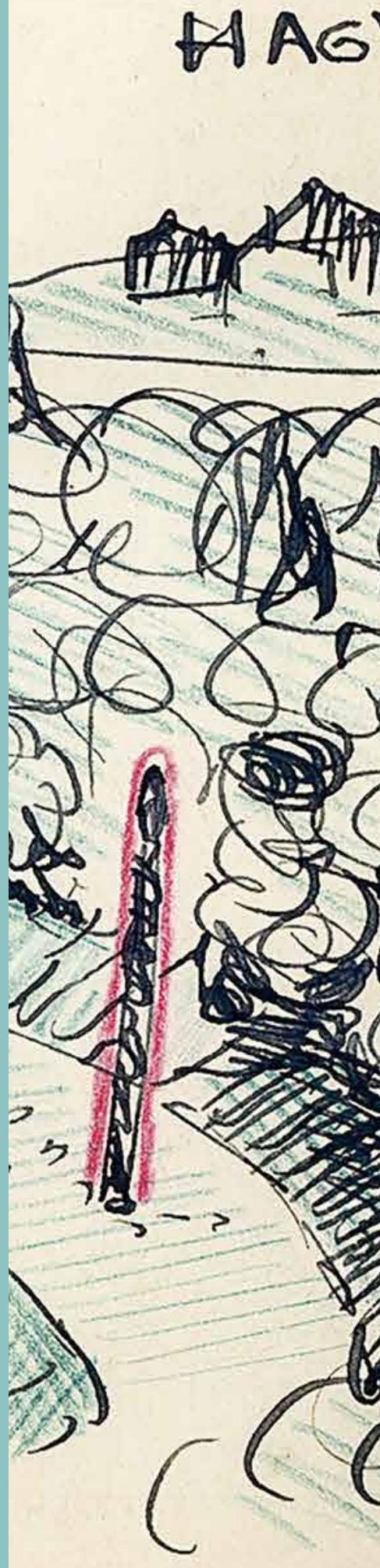